\title{
A toolbox for studying cortical physiology in primates
}

2 Authors: Karam Khateeb ${ }^{1,2}$, Julien Bloch ${ }^{1,2}$, Jasmine Zhou ${ }^{1,2}$, Mona Rahimi ${ }^{2}$, Devon J. Griggs ${ }^{2,3}$,

3 Viktor N. Kharazia ${ }^{4}$, Min N. Le ${ }^{1}$, Ruikang Wang ${ }^{1,5}$, Azadeh Yazdan-Shahmorad ${ }^{1,2,3,6, *}$

4 1. Department of Bioengineering, University of Washington, Seattle, WA 98195, USA

5 2. Washington National Primate Research Center, Seattle, WA 98195, USA

6 3. Department of Electrical and Computer Engineering, University of Washington, Seattle, WA

798195, USA

8 4. Department of Physiology and Center for Integrative Neuroscience, University of California,

$9 \quad$ San Francisco, CA 94143, USA

10 5. Department of Ophthalmology, University of Washington Medicine, Seattle, WA 98195,

11 USA

12 6. Lead Contact

13 *Correspondence: azadehy@uw.edu (AYS) 


\section{Summary}

2 Lesioning and neurophysiological studies have facilitated the elucidation of cortical functions and

3 mechanisms of functional recovery following injury. Clinical translation of such studies is

4 contingent on their employment in non-human primates (NHPs), yet tools for monitoring and

5 modulating cortical physiology are incompatible with conventional NHP lesioning techniques. To

6 address these challenges, we developed a toolbox demonstrated in seven macaques. We introduce

7 the photothrombotic method to induce focal cortical lesions alongside a quantitative model for the

8 design of lesion profiles based on experimental needs. Large-scale $\left(\sim 5 \mathrm{~cm}^{2}\right)$ vascular and neural

9 dynamics can be monitored and lesion induction can be validated in vivo with optical coherence

10 tomography angiography and our electrocorticographic array, the latter of which also enables

11 testing stimulation-based interventions. By combining optical and electrophysiological techniques

12 in NHPs, we can enhance our understanding of cortical functions, investigate functional recovery

13 mechanisms, integrate physiological and behavioral findings, and develop treatments for

14 neurological disorders.

\section{Key Words}

16 Non-human primates, cortex, photothrombotic lesioning, optical coherence tomography

17 angiography, electrophysiology, electrocorticography, electrical stimulation

\section{Introduction}

19 The primate neocortex is responsible for a variety of complex tasks and behaviors, including long-

20 term memory storage, sensory processing, and movement. Historically, both lesioning and

21 neurophysiological studies have been critical for elucidating functions of specific cortical regions

22 (Ferrier, 1876) such as somatosensory (Borich et al., 2015; Brinkman et al., 1985; Gerlai et al., 
1 2000), visual (Humphrey, 1974; Wurtz and Goldberg, 1972), auditory (Heffner and Heffner,

2 1986), and posterior parietal (Murphy et al., 2016; Vallar et al., 1994) cortices. Recently, such

3 strategies have also been employed to investigate mechanisms of plasticity and recovery following

4 injury (Friel et al., 2007; Harrison et al., 2013; Kaeser et al., 2010; Liu and Rouiller, 1999; Nudo

5 and Milliken, 1996; Nudo et al., 1996; Padberg et al., 2010; Pons et al., 1988; Xerri et al., 1998a).

6 The study of these phenomena in non-human primate (NHP) models with strong evolutional

7 relevance to the human cortex is critical for understanding fundamentals of cortical physiology

8 and designing novel clinical treatments for cortical injury.

9 Typical strategies for investigating in vivo NHP cortical physiology include either monitoring or

10 perturbing the native cortical activity then correlating neural activity with behavior. Neural activity

11 is frequently monitored through electrical recording, or calcium imaging, while perturbations

12 include lesioning, electrical stimulation, or optogenetic manipulation (Tremblay et al., 2020).

13 Previously, we developed a large-scale interface enabling optogenetic neuromodulation in concert

14 with simultaneous electrical recording (Ledochowitsch et al., 2015a; Yazdan-Shahmorad et al.,

$152015,2016,2018 \mathrm{a}, 2018 \mathrm{~b})$. However, to the best of our knowledge, there is no single unifying

16 paradigm through which the full spectrum of strategies can be combined for the unhindered

17 investigation of cortical physiology in NHPs. Conventional lesioning techniques lack

18 compatibility with tools for monitoring and modulating cortical physiology and lack flexibility in

19 controlling lesion location and extent. Here, we integrate a versatile focal ischemic lesioning

20 technique with large-scale monitoring of cortical vascular dynamics, and electrophysiological

21 recording and stimulation. Importantly, with large-scale stable optical access, this toolbox can be

22 combined with optical stimulation and imaging techniques such as optogenetics and calcium

23 imaging, respectively. 
1 Commonly utilized NHP cortical lesioning techniques are challenging to employ. One method of

2 lesioning cortex in NHPs is through middle cerebral artery occlusion (Maeda et al., 2005; Virley

3 et al., 2004). Because middle cerebral artery occlusion is used to mimic ischemic stroke as

4 observed in the clinic, the resulting lesions are broad and are constrained only to regions

5 downstream of the middle cerebral artery. Moreover, obtaining access to the middle cerebral artery

6 for occlusion requires complex surgical intervention regardless of the occlusion technique.

7 Similarly, common focal cortical lesioning techniques such as endothelin-1 (Dai et al., 2017; Teo

8 and Bourne, 2014), electrocoagulation (Nudo et al., 2003; Xerri et al., 1998b), ibotenic acid

9 (Kaeser et al., 2010; Liu and Rouiller, 1999), cooling (Brinkman et al., 1985), and aspiration

10 (Heffner and Heffner, 1986; Padberg et al., 2010; Pons et al., 1988) also involve technically

11 challenging surgical procedures that are susceptible to variability across animals. Importantly,

12 these techniques lack compatibility with tools for monitoring cortical physiological dynamics

13 during lesion formation and recovery.

14 When conducting lesion studies in NHPs, it is critical that lesions are validated in vivo for studies

15 involving long-term post-lesion behavioral and physiological assessment. Current lesion

16 validation methods primarily consist of behavioral assessment and post-mortem histological

17 analysis (Kaeser et al., 2010; Liu and Rouiller, 1999; Murata et al., 2008). Methods of in vivo

18 lesion validation, in addition to behavioral assessment, include magnetic resonance imaging (MRI)

19 (Le Friec et al., 2020), or visual assessment of tissue blanching due to devascularization (Nudo

20 and Milliken, 1996; Nudo et al., 1996; Xerri et al., 1998a). While MRI validation can provide both

21 anatomical and physiological assessment of lesion induction, it exhibits poor spatial and temporal

22 resolution (Baran and Wang, 2016). Moreover, visual assessment can result in unreliable

23 estimation of lesion boundaries (Xerri et al., 1998a). The reliance on behavioral and post-mortem 
1 histological analysis alone is deficient of direct anatomical and physiological lesion validation

2 during the lifetime of the animal. Ideally, lesion validation would be accomplished reliably, and in

3 a manner that allows for monitoring of anatomical and physiological changes over the course of

4 recovery.

5 In addition to these techniques, the investigation of cortical neurophysiological dynamics can play

6 an important role in enhancing our understanding of cortical physiology and in developing

7 neurorehabilitative strategies. The monitoring of neurophysiological dynamics in neuroscience is

8 often accomplished through electrical stimulation and recording of neural activity. For example,

9 intracortical microstimulation of primary motor cortex following a lesion has been crucial in

10 elucidating the remapping of cortical somatotopic representations (Nudo and Milliken, 1996; Nudo

11 et al., 1996). In other cortical regions, electrophysiological recordings have also been used to

12 monitor neural activity following lesion induction (Padberg et al., 2010; Schmid et al., 2009). The

13 incompatibility of the lesioning methods used in these studies with recording and stimulation

14 techniques, however, prevents the monitoring of neural activity simultaneously during lesion

15 formation. Ideally, neural activity would be recorded before, during, and after lesion formation,

16 while stimulation-based interventions could be tested after lesioning.

17 In this study, we addressed the technical shortcomings of studying cortical physiology in NHPs.

18 We successfully demonstrate the combination of a photochemical lesion induction technique

19 with a controlled spatial profile, an in vivo lesion validation method with high spatial resolution,

20 and the ability to simultaneously monitor the underlying neural activity and blood flow as lesions

21 form at a large scale $\left(\sim 5 \mathrm{~cm}^{2}\right)$. This toolbox also offers the capability to test stimulation-based

22 interventions. Moreover, the sizes of focal lesions induced with our toolbox are comparable to

23 previously reported focal lesions capable of eliciting behavioral deficits (Murata et al., 2008; 
1 Padberg et al., 2010). Importantly, the tools presented here are compatible with previously

2 established interfaces offering large-scale access for optical and electrical stimulation as well as

3 imaging (Griggs et al., 2021b; Macknik et al., 2019; Yazdan-Shahmorad et al., 2016), providing

4 unparalleled access to the primate cortex. Through this integrative approach, the large-scale

5 monitoring of cortical network activity and reorganization concurrently with vascular dynamics

6 expands the questions and interventions we can explore, profoundly impacting the development

7 of neurorehabilitative therapies.

\section{Results}

9 To address the challenges of studying cortical physiology in NHPs, we developed a toolbox that

10 allows for targeted focal lesioning, large-scale imaging of cortical blood flow, and large-scale

11 electrophysiological recording and stimulation. Our toolbox is comprised of the photothrombotic

12 technique for inducing focal ischemic lesions, optical coherence tomography angiography

13 (OCTA) imaging for in vivo lesion validation, histological validation, a computational model for

14 designing lesion profiles, and electrocorticographic (ECoG) recording during lesion formation.

15 We implemented the photothrombotic technique (Figure 1) in 7 adult macaques (monkeys A-G)

16 and validated the disruption of cortical blood flow in vivo using OCTA imaging as described in

17 the STAR Methods. Lesions were induced by illuminating through an opaque apertured mask

18 (Figure 1A) in a $25 \mathrm{~mm}$ diameter cranial window with an uncollimated white light source

19 following intravenous infusion of the photoactive dye Rose Bengal (Figure 1B). Photoactivation

20 of Rose Bengal in the cerebral vasculature results in the release of reactive oxygen species,

21 subsequent endothelial cell damage, platelet activation, and vascular occlusion due to the

22 formation of thrombi. We tested the effects of a range of aperture diameters (0.5 to $2 \mathrm{~mm})$ and

23 illumination intensities ( 0.1 to $18.6 \mathrm{~mW}$ ) on lesion size. To test these variables, we used an opaque 
1 mask with multiple apertures of various diameters dispersed throughout the cranial window

2 (Supplementary Figure 1). A single uncollimated white light source was used to illuminate through

3 the mask apertures such that the light intensity was highest through centrally located apertures.

4 Through this approach we were able to test a variety of illumination parameters while reducing

5 the number of animal subjects, time under anesthesia, and the number of light sources to practical

6 levels.

$7 \quad$ Validation of Neuronal Cell Loss by Histology and Immunohistochemistry

8 Approximately 4 hours after the end of illumination, animals were perfused with $4 \%$

9 paraformaldehyde (PFA) and the brains were extracted. Prior to histological staining of coronal

10 slices, we observed bright pink areas corresponding with the illuminated regions (Figure 2A),

11 suggesting that the formation of thrombi occluded the vasculature, entrapping the pink-colored

12 Rose Bengal even after perfusion. We then stained coronal sections of the brains for Nissl bodies

13 (Figure 2B,C). In illuminated regions, we observed areas characterized by well-defined borders

14 that lacked Nissl staining, demonstrating cell loss in those regions (Figure 2B,C). We identified

15 these areas as lesions. To test for neuronal cell loss, we performed NeuN staining and observed

16 similarly defined borders in the illuminated regions (Supplementary Figure 2). Depending on

17 aperture sizes and illumination intensities, lesion depths extended through all cortical layers. To

18 gain perspective of the relative locations and sizes of the lesions, we reconstructed the lesions in

19 three-dimensional space using the coronal Nissl-stained sections (Figure 2D-H). With this method,

20 we measured lesion volumes ranging between $1.7 \mathrm{~mm}^{3}$ and $35.4 \mathrm{~mm}^{3}$. We observed 22

21 histologically detected lesions out of 30 illuminated areas across monkeys B-E. Notably, high

22 intensity illumination through centrally located apertures more consistently resulted in

23 histologically detected lesions (lesion volumes ranging from $10.1 \mathrm{~mm}^{3}$ to $31.2 \mathrm{~mm}^{3}$, median of 
$1 \quad 19.6 \mathrm{~mm}^{3}$ ) compared to lower intensity illumination in peripheral apertures (volumes $0 \mathrm{~mm}^{3}$ to

$2 \quad 35.4 \mathrm{~mm}^{3}$, median of $2.9 \mathrm{~mm}^{3}$ ).

3 Optical Coherence Tomography Angiography for Large-Scale Blood Flow Imaging and

4 In Vivo Lesion Validation

5 The extent of optical access afforded by our toolbox enables for the application of large-scale

6 imaging techniques such as OCTA imaging. OCTA is a non-invasive angiographic technique that

7 is capable of imaging functional vascular networks within tissue beds in vivo. OCTA has been

8 demonstrated to detect inflammatory conditions in skin (Deegan and Wang, 2019; Deegan et al.,

9 2018a), eyes (Kashani et al., 2017), and brain (Li et al., 2018; Park et al., 2018). While post-

10 mortem histological analysis is the predominant method of measuring focal lesion size, we

11 introduce OCTA as an in vivo tool to image blood flow in the cortical microvasculature, validate

12 lesion induction, and measure ischemic lesion sizes. We imaged several $9 \mathrm{~mm}$ x $9 \mathrm{~mm}$ cortical

13 regions subjected to photothrombotic ischemia.

14 OCTA imaging 3 hours after illumination revealed clear localized disruptions in cortical blood

15 flow compared to baseline images in the illuminated regions (Figure 3, Supplementary Figure 3),

16 as evidenced by a lack of functional blood vessels in regions interrogated by the photothrombotic

17 illumination. Thus, we demonstrated the ability to validate lesion induction in vivo through OCTA

18 imaging.

19 We then compared OCTA and histological lesion validation modalities (Figure 4A-C). Not all

20 illuminated regions resulted in lesions detected through both modalities. Across monkeys B

21 through E, we illuminated 30 regions to induce photothrombosis. 26 of the illuminated regions

22 were imaged within the OCTA field of view (FOV), of which 16 lesions were detected through 
1 OCTA imaging. 15 of the 16 OCTA-detected lesions were later confirmed histologically with one

2 lesion undetected histologically. Similar to histology, illumination through centrally located

3 apertures corresponding with higher light intensities more consistently resulted in lesions detected

4 with OCTA compared to peripheral apertures with lower light intensities. Additionally, 8 of the

510 imaged regions in which no lesions were detected in OCTA images corresponded with regions

6 occupied by large blood vessels. Of those 8 regions, only 3 resulted in lesions detected through

7 histology (3.7 to $17.6 \mathrm{~mm}^{3}$ ). Of the 22 histologically detected lesions, 15 were detected through

8 OCTA. Histologically detected lesions within the OCTA FOV that were not detected in OCTA

9 images (4 lesions) were likely a result of illumination of large vessels (3 lesions), or the lesion may

10 have been too small to be detected via OCTA imaging (1 lesion, $2.83 \mathrm{~mm}^{3}$ ). Notably, OCTA

11 detection of lesions was unsuccessful in all instances in which the illuminated region coincided

12 with large blood vessels, regardless of histological detection (Supplementary Figure 4). Because

13 of the inconsistencies of large vessel illumination due to differences in optical and biological

14 properties, we excluded data corresponding with large vessel illumination from further analysis.

15 We then quantitatively assessed OCTA-measured lesion diameters as in vivo proxy measurements

16 of histologically measured lesion diameters (Figure 4B). We observed a positive linear relationship

17 between OCTA-measured lesion diameters and histologically measured lesion diameters, with an

18 r-squared value of $0.81(\mathrm{p}=3.9 \mathrm{e}-7$; Figure 4B). Because OCTA imaging provides depth

19 information only down to about $600 \mu \mathrm{m}$, we assessed the correlation between histologically

20 measured lesion depths and OCTA-measured lesion diameters (Figure 4C). We observed a

21 positively correlated linear relationship between histological lesion depth and OCTA-measured

22 lesion diameter with an r-squared value of $0.50(\mathrm{p}=0.0011$; Figure $4 \mathrm{C})$. Interestingly, we also

23 observed a significant positive correlation between both histologically measured lesion depths and 
1 diameters (0.65 r-squared; $p=5.3 \mathrm{e}-6$; Supplementary Figure 4). Importantly, our results

2 demonstrate that OCTA-measured lesion diameter is a strong predictor of histologically measured

3 diameters.

\section{Effect of Illumination Parameters on Lesion Size}

5 We examined the combined contributions of both aperture diameter and illumination intensity as

6 predictors of lesion size through multivariate linear regression analysis (Figure 4G-L,

7 Supplementary Table 1). As the Beer-Lambert law (Bouguer, 1729) predicts an optical path length

8 with logarithmic relation to light intensity, we used the log-transformed light intensity as a

9 predictor. We found that a model accounting for both sets of illumination parameters accounts for

10 a large portion of the variance in OCTA-measured diameters with an $\mathrm{r}$-squared value of $0.85(\mathrm{p}=$

11 6.5e-7; Figure 4D,G,J). In predicting histologically measured lesion diameters, the combined

12 illumination parameters yielded an $r$-squared value of $0.81(\mathrm{p}=1.8 \mathrm{e}-7$; Figure 4E,H,K). For the

13 prediction of histologically measured lesion depths based on both aperture diameter and light

14 intensity, we observed an r-squared value of $0.49(\mathrm{p}=0.0017$; Figure 4F,J,L). Therefore, both

15 illumination parameters serve as significant determinants of lesion size, and the consideration of

16 their combined effects is critical for predicting lesion sizes.

17 Prediction of Lesion Size by Simulation of Light Propagation through Cortical Tissue

18 To extend the predictability of lesion sizes based on illumination parameters beyond those tested

19 in this study, and to be able to predict both lesion diameter and depth with a single biophysically

20 inspired model, we developed a simulation-based computational model. Photothrombotic lesions

21 arise due to thrombi formation and subsequent cell death mediated by the topography of the

22 underlying microvasculature. As such, we developed a two-stage modeling process in which we

23 simulated photons penetrating brain tissue (Figure 5S) to generate a profile of the spatial fluence 
1 distribution (Figure 5B) and transformed fluence contours to recreate the lesion shapes observed

2 through histology (Figure 5C,D).

3 To model the illuminated cortical tissue, we constructed a virtual optical illumination and medium

4 model of our experiments, consisting of a light source and mask, artificial dura, gray matter, and

5 white matter (Figure 5A). The entire volume spanned a size of $8 \mathrm{~mm} \times 8 \mathrm{~mm} \times 5.6 \mathrm{~mm}$ (width $\mathrm{x}$

6 width $\mathrm{x}$ height) and was constructed in the Monte Carlo eXtreme (MCX) software (Fang and Boas,

7 2009). In this volume, uncollimated light exiting our compound light source (see STAR Methods)

8 passes through the artificial dura, gray matter, and white matter. By simulating stochastic photon

9 transmission through this medium based on different illumination parameters, we can obtain

10 spatial fluence distributions over the volume. As the photothrombotic lesioning method relies on

11 the interaction between light and Rose Bengal to occlude blood flow, this spatial distribution can

12 describe the effect of illumination parameters on lesion induction.

13 The optical properties of gray and white matter scattering and absorption coefficients had wide

14 ranging values previously reported in literature (Gottschalk, 1992; Yaroslavsky et al., 2002).

15 Therefore, we ran a grid-search of Monte Carlo simulations of 1 million photons propagating

16 through a virtual medium with these optical properties within the reported ranges to find a best fit

17 to our data. For each set of optical properties in the grid we ran a simulation for our experimental

18 aperture diameter and illumination intensity combinations. A spatial fluence distribution threshold

19 was then identified (Figure 5B) for each set which maximized the overlap (see Equation 2,

20 Methods) between the simulated lesions and the corresponding lesions obtained through histology.

21 Using this method, we identified the best optical properties as a gray matter absorption coefficient

22 of $0.395 \mathrm{~mm}^{-1}$, gray matter scattering coefficient of $53.6 \mathrm{~mm}^{-1}$, white matter absorption coefficient 
1 of $0.09 \mathrm{~mm}^{-1}$, white matter scattering coefficient of $54.066 \mathrm{~mm}^{-1}$, and a spatial fluence distribution

2 threshold for lesion induction of $19.9 \mu \mathrm{W} / \mathrm{mm}^{2}$.

3 From the identified best-matching light simulations we quantified the degree to which they predict

4 lesion shapes and sizes. We observed that while the simulation results qualitatively align with the

5 lesion profiles, the light simulation alone was not adequate for explaining the observed lesions. By

6 using the maximal depth and average diameter of individual fluence threshold contours of the

7 simulation as predictions of their corresponding lesion profiles, we observed that the contours

8 resulted in an $\mathrm{r}$-squared value of -0.04 for lesion depth and 0.32 for diameter. These results indicate

9 that the extent of induced photothrombotic ischemic lesions in vivo is not simply a function of the

10 fluence profile in the cortex but is likely also governed by biological factors such as vascular

11 topography.

12 We therefore incorporated a scaling process to transform the light intensity contours to the

13 biological lesion profiles observed through histology (Figure 5C,D). We observed that the

14 diameters from the simulation scaled linearly to match the diameters observed from histology, with

15 a scaling factor of 0.726 yielding an r-squared value of 0.82 (Figure 5E). The depth did not scale

16 linearly well, however, as the best linear scaling of depth yielded an r-squared value of 0.13

17 (Supplementary Figure 5). From examining the residuals, we observed that square root

18 transforming the depth before linear scaling would yield a more accurate prediction

19 (Supplementary Figure 5). Indeed, by square root transforming the depth and scaling by a factor

20 of 1.6969 we obtained an r-squared value of 0.41 (Figure 5F). Interestingly, although our model

21 was developed based on histologically derived lesion contours, the resulting model-derived lesion

22 diameters were also highly predictive of OCTA-measured lesion diameters with an r-squared value 
1 of 0.60 (Figure $5 \mathrm{G}$ ). Thus, our quantitative model is able to accurately predict lesion shape,

2 diameter, and depth.

3 Large-Scale Neurophysiological Recording Before, During, and After Lesion Formation

4 A valuable tool for studying neurophysiological dynamics following cortical lesioning in vivo is

5 the ability to record neural activity. In monkeys D and E we used a semi-transparent 32-electrode

6 ECoG array to record neural activity in sensorimotor cortex during the formation of a lesion

7 (Figure 6A). The transparency of the ECoG array allowed for the induction of a lesion by

8 illuminating through the array. For monkeys D and E, only a single region in the center of the

9 cranial window was illuminated with a $1.5 \mathrm{~mm}$ diameter aperture to induce photothrombosis

10 unilaterally. Similarly, the transparency also enabled imaging of the underlying cortical

11 microvasculature by OCTA to confirm the presence of the lesion (Figure 6A,B). We recorded

12 baseline local field potentials (LFPs) for 30 minutes, followed by 30 minutes during the

13 illumination period, and for up to three hours following illumination.

14 To measure the effect of lesioning on neural activity levels, we analyzed the changes in gamma

15 band $(30-59 \mathrm{~Hz})$ signal power from LFPs recorded during the formation of the lesions. The average

16 gamma power was calculated over the course of 30 minutes of baseline activity, and between 2.5

17 to 3 hours after the illumination period (Figure 6C). We calculated the change in power across the

18 array and identified regions with statistically significant reductions in gamma power as

19 corresponding to the lesioned areas (left-tailed paired t-test, family-wise error rate $<0.001$ ). As

20 expected, channels near the illuminated region exhibited significant decreases in gamma power in

21 both monkeys D and E compared to more distant regions (one-way ANOVA, monkey D: $p=5.2 \mathrm{e}-$

22 9; monkey E: p = 1.0e-5; Figure 6C,D). Notably, we demonstrated the capability to monitor

23 neurophysiological dynamics during lesion formation. Here, the reduction in gamma power was 
1 observed early during the illumination period across all channels, after which distant channels

2 returned to baseline levels while channels near the illuminated region remained suppressed (Figure

$36 \mathrm{E})$.

4 Electrical Stimulation Enables Modulation of Perilesional Neural Activity

5 The ability to stimulate neural activity in the same paradigm that allows for cortical lesioning is

6 critical for the development of stimulation-based interventions. Similar to monkeys D and E,

7 monkeys $\mathrm{F}$ and $\mathrm{G}$ underwent unilateral photothrombotic lesioning through a $1.5 \mathrm{~mm}$ diameter

8 aperture with ECoG recording of activity. We then stimulated through a single perilesional channel

9 in the ECoG array one hour following illumination for a duration of about one hour, after which

10 we continued recording for approximately one hour. Stimulation occurred in six 10-minute blocks

11 separated by 2-minute recording blocks (Figure 7A). To monitor network dynamics following

12 photothrombotic lesioning and during stimulation, we calculated the change in power with respect

13 to baseline in high gamma $(60-150 \mathrm{~Hz})$ and theta $(4-7 \mathrm{~Hz})$ bands. We observed an overall decrease

14 in high gamma power across the array throughout stimulation in monkeys $\mathrm{F}$ and $\mathrm{G}$. This was

15 different from what we observed in non-stimulated monkeys D and E, in which some channels

16 were increasing in high gamma power at equivalent time points (Figure 7B-D). In both stimulated

17 and unstimulated animals, we observed an overall decrease in theta power across the array (Figure

18 7B-D). Importantly, we demonstrated the ability to electrically stimulate neural activity in the

19 context of our toolbox along with the ability to monitor subsequent effects on network neural 20 dynamics. 


\section{Discussion}

2 The presented toolbox offers significant advantages for investigating critical questions regarding

3 cortical physiology. Here, we demonstrate the photothrombotic technique for inducing focal

4 ischemic lesions in NHP cortex. The extent of subsequent neuronal cell loss was later confirmed

5 histologically. Moreover, we developed a quantitative model for predicting photothrombotic lesion

6 sizes based on illumination parameters. Through this model, lesions can be designed in accordance

7 with experimental needs. We also present OCTA imaging as a tool for observing vascular

8 dynamics and validating lesion induction in vivo. Additionally, our ECoG array allows for the

9 investigation of neural dynamics before, during, and after lesion formation. Critical for developing

10 therapeutic interventions, we also establish the ability to test stimulation-based strategies with our

11 ECoG array. Although not demonstrated here, the toolbox allows for experimentation at clinically

12 relevant time scales when employed in NHPs in contrast to rodent studies.

13 The photothrombotic technique allows for the induction of targeted focal ischemic lesions in any

14 cortical region of interest. Using the photothrombotic technique, we can achieve greater spatial

15 control over lesion induction in comparison with commonly employed middle cerebral artery

16 occlusion models (Sommer, 2017). The ability to induce consistent smaller, targeted lesions can

17 allow for the induction of lesions to impact specific cortical functions without risking serious

18 injury or mortality compared to middle cerebral artery occlusion (Wu et al., 2016). In rodents, the

19 photothrombotic technique has enabled the study of local cortical remapping in sensorimotor

20 cortex (Harrison et al., 2013). Larger lesions can also be induced with areas as large as the applied

21 cranial window. Moreover, middle cerebral artery occlusion (Maeda et al., 2005),

22 electrocoagulation (Friel et al., 2007; Nudo et al., 1996; Xerri et al., 1998b), and aspiration methods

23 (Padberg et al., 2010) of cortical lesioning require complex surgical intervention and are performed 
1 in an invasive manner. In contrast, photothrombotic lesion induction only requires optical access

2 to cortex. With the aid of our predictive computational model, lesions can be designed based on

3 experimental needs. However, as is typical with optical techniques, photothrombotic lesion

4 induction with minimal invasiveness is limited to optically accessible regions. To access

5 subcortical areas, more invasive approaches can be used, such as the use of penetrating optical

6 fibers.

7 Here, we demonstrated the use of OCTA to image large-scale cortical vascular dynamics and to

8 validate the presence of lesions in vivo prior to post-mortem histological analysis. The ability to

9 validate lesion induction in vivo is critical for long-term NHP studies. To our knowledge, this is

10 the first time OCTA imaging has been employed to image macaque cortical vasculature to detect

11 the presence of ischemic lesions. We observed strong correlations between OCTA-measured

12 lesion diameters and histologically measured lesion sizes, suggesting that OCTA imaging can be

13 used to reliably measure lesion sizes in vivo. Unlike MRI, OCTA imaging enables monitoring of

14 vascular dynamics with greater spatial resolution and without the need for contrast agents, though

15 with limited depth penetration. Moreover, the optical access afforded by our toolbox allows for

16 the use of other optical validation methods, including laser doppler flowmetry (Jiang et al., 2006).

17 However, not all illuminated regions resulted in lesions detected via OCTA imaging or histology.

18 This may be due to the coincidence of those regions with large blood vessels, in which light

19 penetration through smooth muscle and endothelial cell layers and large lumen diameters

20 prevented the complete occlusion of the vessel. Conversely, successful occlusion of a large vessel

21 may also induce a larger lesion than expected due to the greater extent of downstream or upstream

22 regions. For example, illumination of a larger vessel resulted in the widest lesion observed across

23 our experiments despite the small aperture of $0.5 \mathrm{~mm}$ (Figure 4A, monkey $\mathrm{C}$ left hemisphere, 
1 Supplementary Figure 4). Therefore, illumination of large vessels should be avoided to ensure

2 successful and predictable lesion induction. Moreover, there was one instance in which a lesion

3 was observed via OCTA imaging but was undetected through histology (Figure 4A, monkey B left

4 hemisphere). This may be due to the superficial nature of the lesion such that it was difficult to

5 detect histologically. Consequently, the small size of the lesion may have enabled collateral blood

6 flow to mitigate damage, a phenomenon which has been previously reported in rodent parietal

7 cortex following photothrombotic lesioning of surface vasculature (Schaffer et al., 2006).

8 Moreover, we demonstrated the ability to monitor underlying neurophysiological dynamics with

9 the ECoG array before and during the development of a lesion for up to three hours. The

10 combination of OCTA imaging and the semi-transparent ECoG array allows for the induction and

11 validation of photothrombotic lesions, the identification of electrodes corresponding with ischemic

12 areas, and the investigation of network physiology and dynamics. Additionally, OCTA imaging

13 and ECoG recording can be used to investigate neurovascular coupling at higher spatial and

14 temporal resolution than functional MRI (fMRI). However, unlike fMRI which allows for online

15 monitoring of neurovascular coupling, OCTA imaging requires post hoc offline analysis. The

16 transparency of the array also allows for the incorporation of other optical techniques such as

17 optogenetics and calcium imaging. Simultaneous ECoG recording and optical coherence

18 tomography in combination with optogenetic stimulation has been previously accomplished in

19 mice (Chin-Hao Chen et al., 2020). We demonstrated the ability to test electrical stimulation-based

20 interventions as presented here and in a previous publication (Griggs et al., 2021a), in extension

21 of prior post-lesion stimulation studies (Nudo and Milliken, 1996; Nudo et al., 1996). While we

22 demonstrated the use of this toolbox during acute lesioning, these same tools can be implemented

23 for long-term studies in combination with a chronically implanted cranial window such as our 
1 previously published optogenetic interface (Griggs et al., 2019; Khateeb et al., 2019a; WKS et al.,

2 2020; Yazdan-Shahmorad et al., 2015, 2016), which exhibits the same spatial scale. In such

3 studies, new lesions can be created or later enlarged without the need for additional surgical

4 intervention, as would be required with the commonly employed electrocoagulation and cortical

5 aspiration methods of focal cortical lesioning.

6 We also developed a computational model to predict the shape and scale of photothrombotic

7 lesions. Our regression results and Monte Carlo simulation-based results together allow for

8 planning of precise lesions with a variety of illumination parameters, informed by previous

9 literature and validated against our experimental results. Importantly, through our grid search, we

10 identified the optimal optical properties of cortical tissue to accurately predict lesion sizes. It is

11 important to note, in our experiments we did not vary illumination time across different lesions,

12 with an illumination time based on previous photothrombotic studies (Gulati et al., 2015).

13 Therefore, our computational model was constructed based on lesions induced with consistent

14 illumination times. However, our model may be modified in future studies to predict lesions

15 induced with different illumination times to account for differences in total energy that would be

16 delivered to induce Rose Bengal photoactivation. There are also notable assumptions which are

17 applied to our model. First, we assume a consistent gray matter depth at $2.5 \mathrm{~mm}$, whereas in reality,

18 the depth of gray matter is variable across cerebral cortex, which can affect lesion depth

19 predictions. Moreover, the final step of our model involves scaling the profiles from probability

20 maps to better reconstruct the profiles of our histologically detected lesions. This was done to

21 account for the topography of the cortical vasculature (Figure 5B-D). The depth-wise upscaling of

22 our model contours is likely due to the perpendicular orientation of penetrating arterioles and

23 venules relative to the cortical surface (Gould et al., 2017). The diameter-wise downscaling can be 
1 ascribed to the redistribution of blood flow in vessels downstream of an occluded vessel to preserve

2 perfusion in these downstream regions (Schaffer et al., 2006), thus potentially reducing the extent

3 of ischemic damage.

4 While the presented tools offer great advantages for investigating physiological dynamics in the 5 context of a cortical lesion, there are limitations that can be addressed in later studies. As discussed

6 earlier, the reliance on optical access for lesion induction limits potential regions of interest to 7 superficial brain structures such as cerebral cortex without compromising neural tissue with 8 penetrating optical probes. Additionally, while we developed a model to accurately predict lesion

9 sizes and shapes based on illumination parameters, variations in the induced lesions are likely a 10 result of differences in local optical properties in addition to vascular topographies. Moreover, the

11 ECoG array presented here exhibits limited spatial resolution with 32 opaque electrodes across an

12 area of approximately $314 \mathrm{~mm}^{2}$ and an electrode pitch on the order of millimeters. With an array

13 with more electrodes in a more compact arrangement, greater spatial resolution can be achieved.

14 Additionally, while the medium in which in the electrodes are embedded is transparent, the

15 electrodes themselves are opaque, which can occlude optical penetration of cortical tissue for

16 OCTA imaging and photothrombotic lesion induction. Such a concern can be addressed through

17 the use of transparent indium tin oxide electrodes (Ledochowitsch et al., 2015b). While our current

18 ECoG array limits use to LFP recordings, smaller electrodes would allow for the recording of

19 multi-unit activity as has been previously demonstrated (Khodagholy et al., 2015). Furthermore,

20 the potential to induce post-lesion behavioral effects are not addressed in this study. However, the

21 successes of previous macaque studies (Murata et al., 2008; Padberg et al., 2010) with focal lesions

22 of sizes similar to those reported here provide encouraging evidence of the potential to induce

23 behavioral deficits with the photothrombotic technique. 
1 Together, these tools can be used to answer critical questions in neuroscience and drive studies of

2 cortical physiology and related disorders such as stroke and traumatic brain injury. Importantly,

3 our toolbox enables the perturbation of the brain and simultaneous monitoring of physiological

4 dynamics over time. The combination of the presented tools can be used to evaluate vascular

5 dynamics, neural dynamics, and neurovascular coupling in the context of either cortical lesioning,

6 stimulation, or both. Although our toolbox is demonstrated here in an acute setting, the same tools

7 are capable of being used in chronic experiments. Such a capability would allow for the monitoring

8 of the behavioral and physiological effects of lesioning and subsequent recovery over time, in

9 combination with testing stimulation-based interventions. Furthermore, the compatibility of our

10 toolbox with optical tools such as optogenetics can enable the comparison of temporary and

11 permanent cortical lesioning techniques. Importantly, long-term studies employing our toolbox

12 can address key questions regarding cortical functions and drive the development of future

13 rehabilitative therapies for stroke, traumatic brain injury, and other relevant neurological disorders.

\section{Acknowledgements}

15 We thank Toni Haun, William Ojemann, Evelena Burunova, Stephen Philips, Warren Han, Zhaojie

16 Yao, Teng Liu, Shaozhen Song, Sandi Thelen, Christopher English, Dean Jeffrey, and Britni Curtis

17 for their help with animal surgeries and experimentation. We also thank the Horwitz and Buffalo

18 labs for their shared expertise on histological analysis, and the Ganguly lab for their expertise on

19 the photothrombotic technique. This work was supported by the Eunice Kennedy Shriver National

20 Institute of Child Health \& Human Development of the National Institutes of Health

21 (K12HD073945, AY), the National Institute of Neurological Disorders and Stroke of the National

22 Institute of Health (1R01NS116464-01, AY, JZ), the National Eye Institute (R01EY028753,

23 RKW), the Washington National Primate Research Center (P51 OD010425), the University of 
1 Washington Royalty Research Fund (AY, KK), the National Science Foundation Graduate

2 Research Fellowship (KK), the Big Data for Genomics and Neuroscience Training Grant (JB), the

3 Weill Neurohub (JZ), and the Center for Neurotechnology (EEC-1028725, DG).

\section{Author Contributions}

$5 \quad \mathrm{KK}$ and JB drafted the manuscript. AY conceptualized the study. AY and KK designed the experiments. AY, KK, DG, MR, JB, JZ, and VK performed the experiments. ML and RKW

7 performed the optical imaging setup and data collection. KK, JZ, MR, JB, and ML performed data 8 analysis. All authors revised and edited the manuscript.

\section{Declaration of Interests}

10 RKW discloses intellectual property owned by the Oregon Health and Science University and the 11 University of Washington. He is a consultant to Carl Zeiss Meditec.

12 The remaining authors declare no disclosures.

13 Figure Legends

A
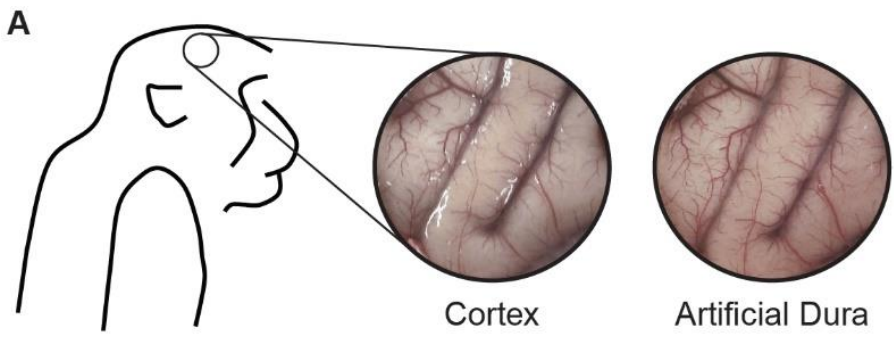
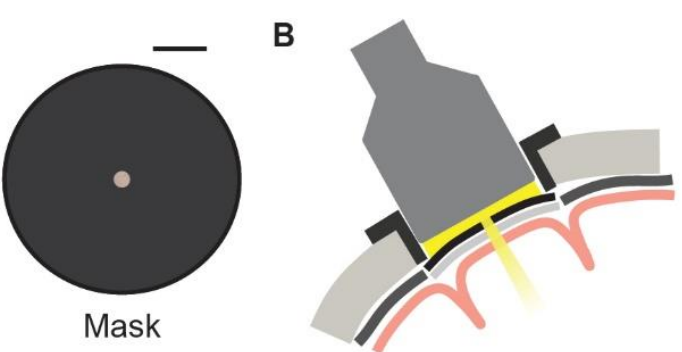

15 Figure 1. Schematic of photothrombotic technique application to induce focal cortical ischemic

16 lesions. (A) Following a $25 \mathrm{~mm}$ diameter circular craniotomy, a thin, transparent artificial dura

17 was placed over the exposed cortical surface. Next, an apertured mask was placed on top of the 
1 artificial dura. Scale bar is $5 \mathrm{~mm}$. (B) Coronal schematic of light illumination through the apertured

2 mask following intravenous Rose Bengal infusion. Portions of this figure have been adapted for

3 inclusion in this manuscript from Khateeb et al., 2019 with permission.

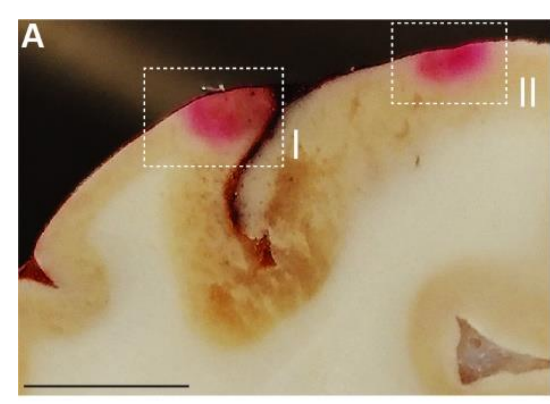

D

$\mathbf{F}$

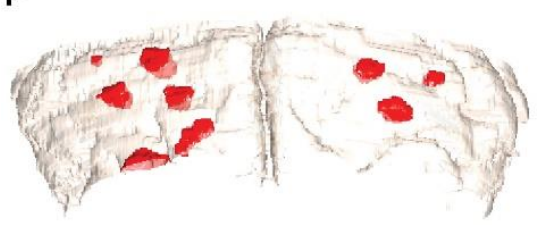

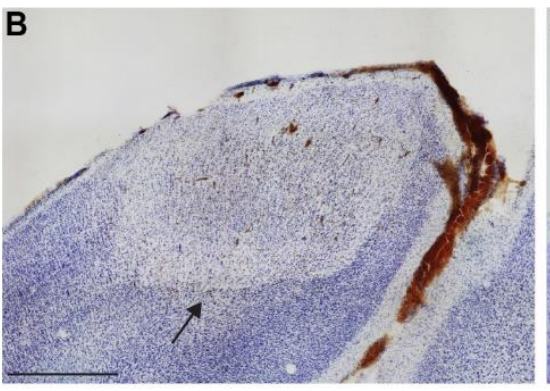

E

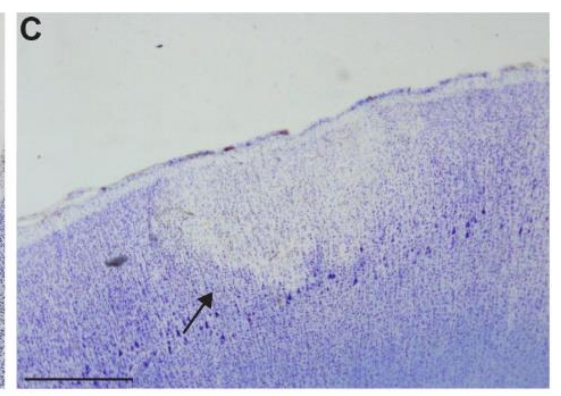

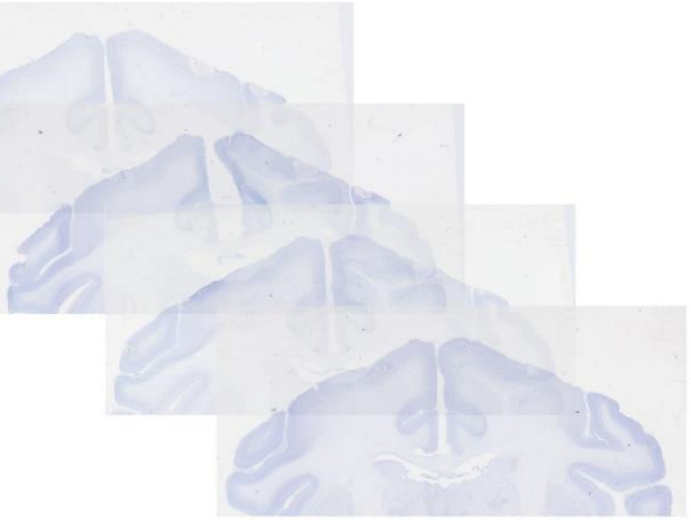

G

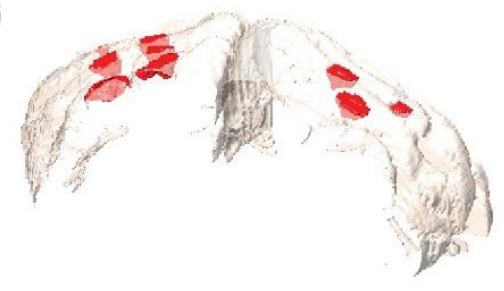

H

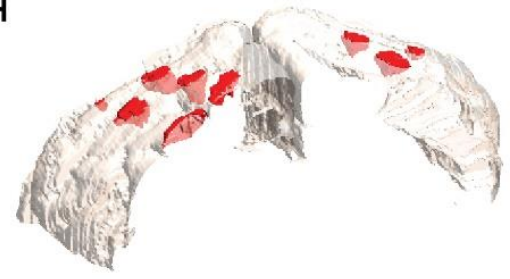

Figure 2. Histological lesion validation and reconstruction. (A) Unstained coronal section from

6 monkey A. Pink regions corresponding with illuminated regions highlighted in boxes I and II

7 indicate the presence of Rose Bengal entrapped in the cortical microvasculature. Scale bar is 5

$8 \mathrm{~mm}$. (B,C) Coronal Nissl-stained slice adjacent to the slice shown in panel (A), showing cell loss

9 in the region encapsulated in box I (B) and II (C) and indicated by the black arrows. Scale bars are

$101 \mathrm{~mm}$. (D,E) Reconstruction of induced lesions in 3D space was done by first co-registering 
1 coronal Nissl-stained slices (D), followed by identification of lesion boundaries (E). (F-H), 3D

2 reconstruction of lesions in monkey $\mathrm{C}$ from three different angles. Portions of this figure have been

3 adapted for inclusion in this manuscript from Khateeb et al., 2019 with permission.

A

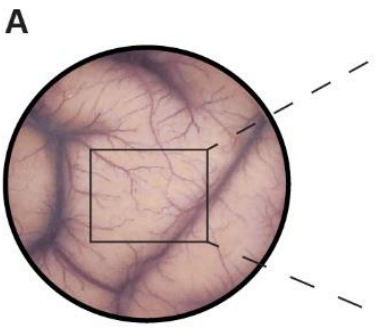

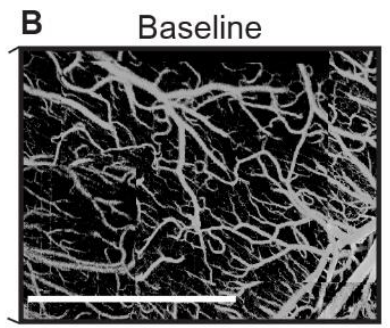
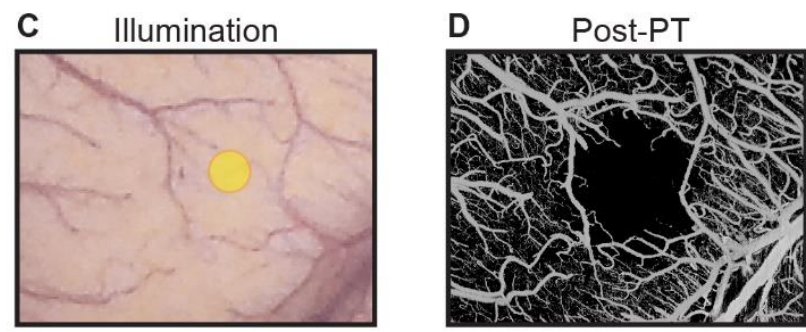

5 Figure 3. Optical coherence tomography angiography (OCTA) validation of lesion induction. (A)

6 Surface of sensorimotor cortex through an artificial dura in monkey C. (B) OCTA imaging of the

7 rectangular area prior to lesion induction. Scale bar is $5 \mathrm{~mm}$. (C) Illumination of a cortical region

8 of interest indicated by the yellow circle following intravenous Rose Bengal infusion. (D) OCTA

9 imaging 3 hours post-photothrombosis. Portions of this figure have been adapted for inclusion in

10 this manuscript from Khateeb et al., 2019 with permission. 
A

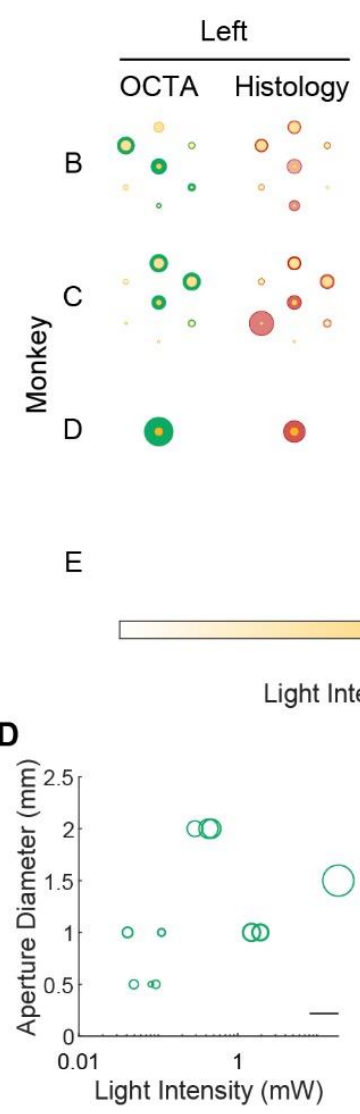

G

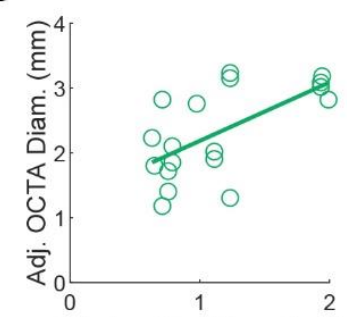

Adj. Aperture Diam. (mm)

J

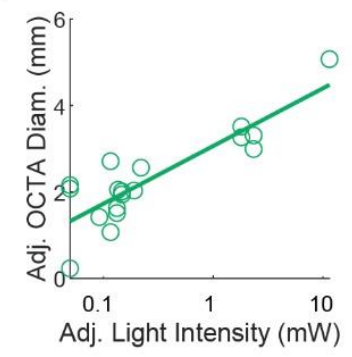

$\frac{\text { Right }}{\text { OCTA Histology }}$

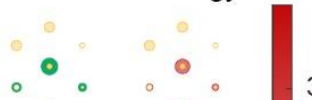

$0^{\circ} \circ$

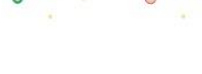

E

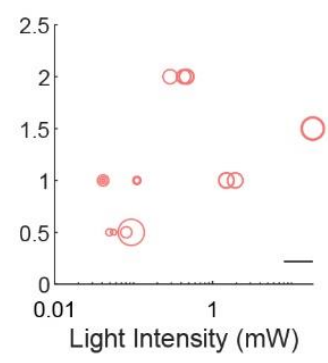

H

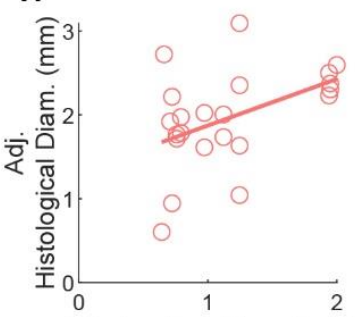

Adj. Aperture Diam. (mm)

K

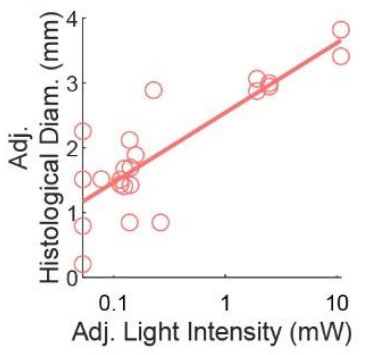

B

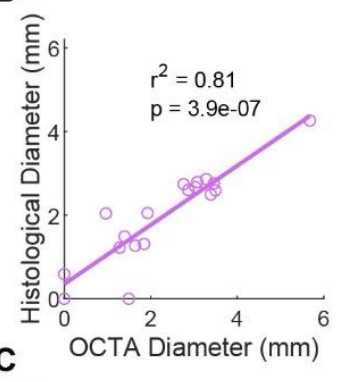

2

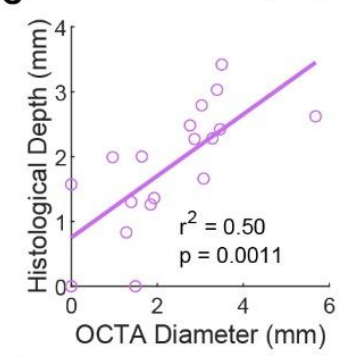

F

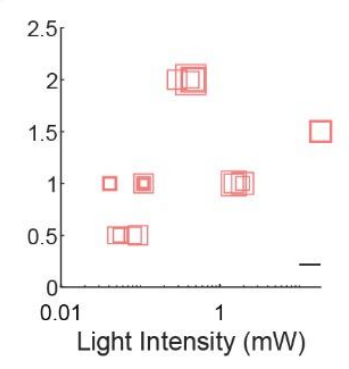

I

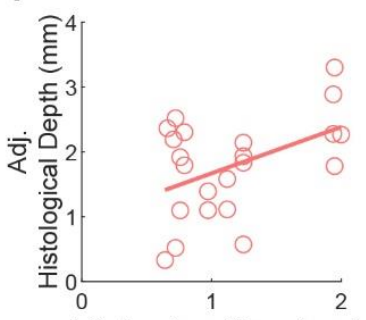

Adj. Aperture Diam. (mm)

L

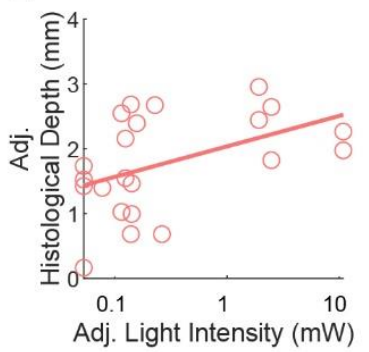

2 Figure 4. Comparison of OCTA and histological lesion validation and effect of illumination

3 parameters. (A) Schematic demonstrating the OCTA-measured lesion diameters (green circles) in 
1 the illuminated regions (yellow circles) of both hemispheres for monkeys B-E. The shading of the

2 yellow circles indicates illumination intensity. The histologically measured lesion diameters are

3 also shown (pink circles), with the shading indicating lesion depth. (B) OCTA-measured and

4 histologically measured lesion diameters were highly correlated (0.81 r-squared, $\mathrm{p}=3.94 \mathrm{e}-7)$. (C)

5 OCTA-measured lesion diameters also correlated with histologically measured lesion depth $(0.50$

6 r-squared, $\mathrm{p}=0.0011$ ). (D) Combined effect of aperture diameter and light intensity on OCTA-

7 measured lesion diameter. Circular marker diameters represent OCTA-measured lesion diameter.

8 Scale bar is $5 \mathrm{~mm}$. (E) Combined effect of aperture diameter and light intensity on histologically

9 measured lesion diameter, where the diameter of the circular markers represents histologically

10 measured lesion diameter. Scale bar is $5 \mathrm{~mm}$. (F) Combined effect of aperture diameter and

11 illumination intensity on histologically measured lesion depth. The width of the square markers

12 denotes histologically measured lesion depth. Scale bar is $2 \mathrm{~mm}$. (G-L), Partial regression plots

13 demonstrating the effects of aperture diameter and light intensity via a multivariate linear model

14 on OCTA-measured lesion diameters $(0.85$ r-squared, $\mathrm{p}=6.5 \mathrm{e}-7)(\mathrm{G}, \mathrm{J})$, histologically measured

15 diameters $(0.81 \mathrm{r}$-squared, $\mathrm{p}=1.8 \mathrm{e}-7)(\mathrm{H}, \mathrm{K})$, and histologically measured depths $(0.49 \mathrm{r}$-squared,

$16 \mathrm{p}=0.0017)(\mathrm{I}, \mathrm{L})$. In panels $\mathrm{G}-\mathrm{L}$, the partial regressions are shown with the adjusted variables. 


\section{A}
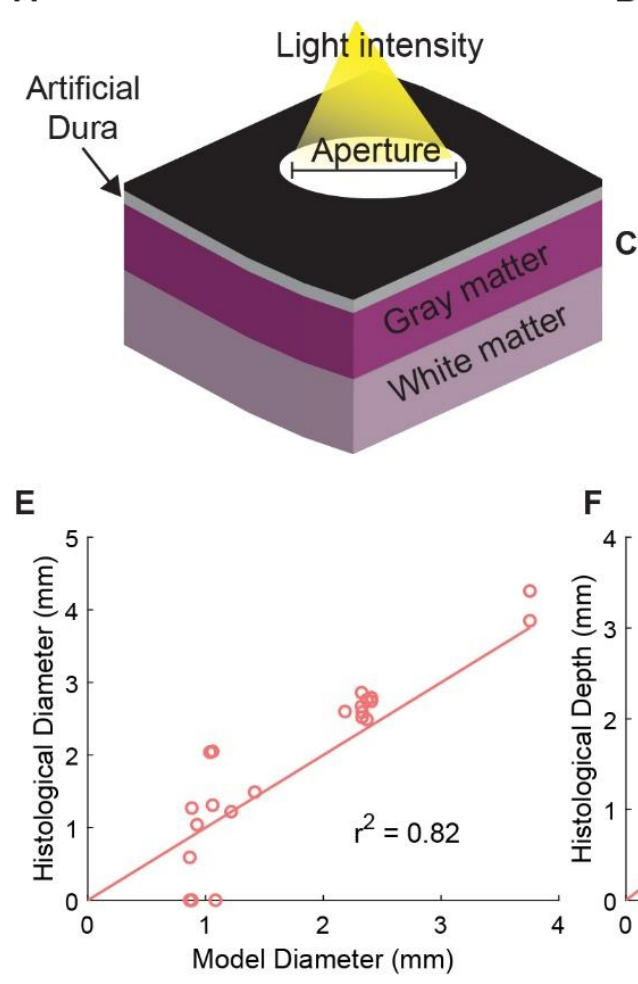

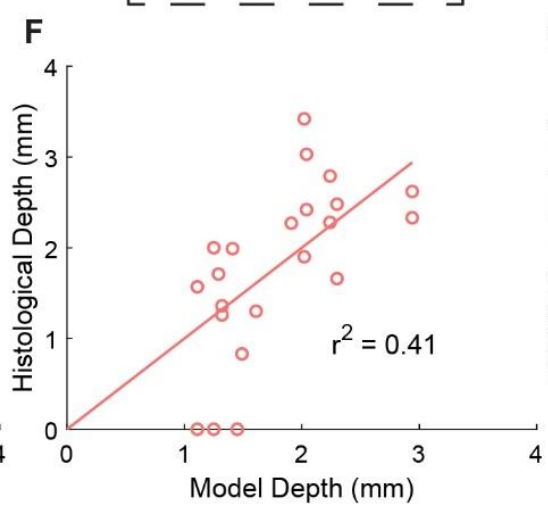

B
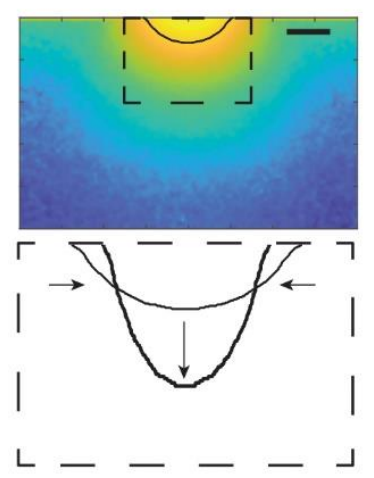

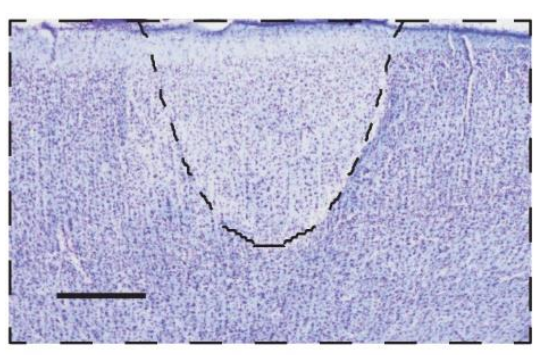

G

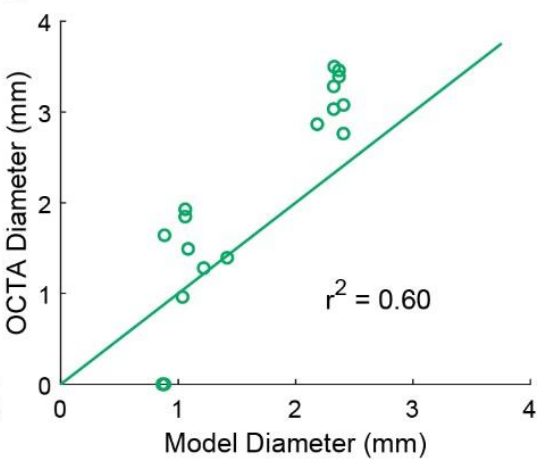

Figure 5. Prediction of lesion size by simulation of light propagation through cortical tissue. (A)

3 Schematic of simulated cortical volume with our experimental setup. An uncollimated light beam passes through an aperture and a transparent artificial dura $(0.5 \mathrm{~mm}$ thick), into gray and white matter of a virtual cortical medium. Gray matter thickness is $2.5 \mathrm{~mm}$. (B) A contour is identified

6 from the light profile matching the light intensity threshold $\left(19.9 \mu \mathrm{W} / \mathrm{mm}^{2}\right)$ most closely matching

7 the lesions. Scale bar is $100 \mu \mathrm{m}$. (C) The light intensity contour is scaled to generate a biological

8 lesion contour. Scaling factors were obtained through regression on our dataset of simulated lesion

9 dimensions and corresponding histologically measured lesions. (D) Predicted lesion contour

10 overlayed on a coronal Nissl-stained slice of a corresponding lesion from monkey B. Scale bar is

$1150 \mu \mathrm{m}$. (E-G), Modeled lesions accurately predict histologically measured lesion diameters $(0.82$

12 r-squared) (E) depths (0.41 r-squared) (F), and OCTA-measured diameters (0.60 r-squared) (G). 
A

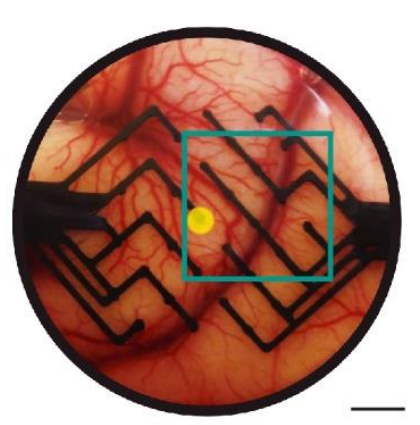

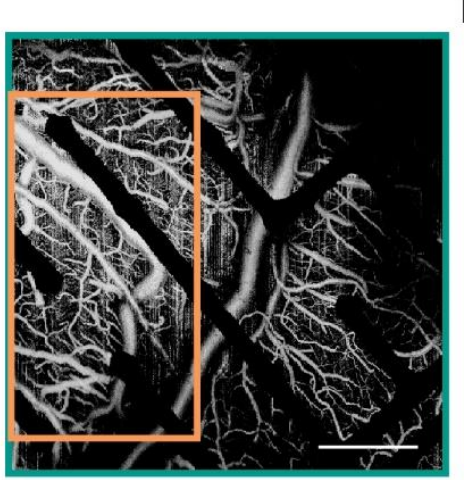

B

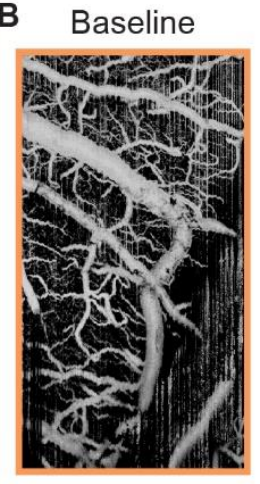

C
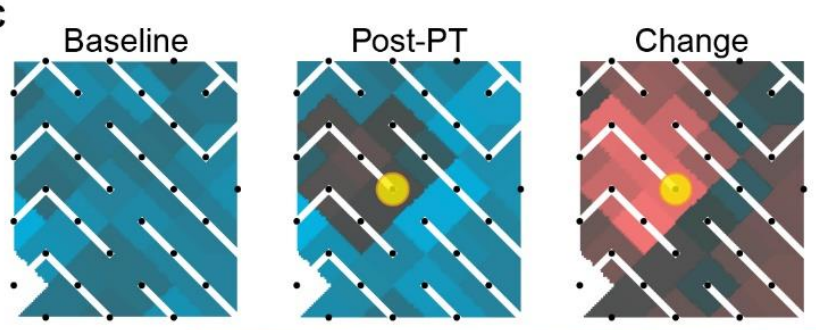

$-0.1$

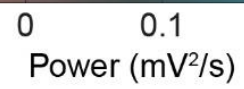

Post-PT

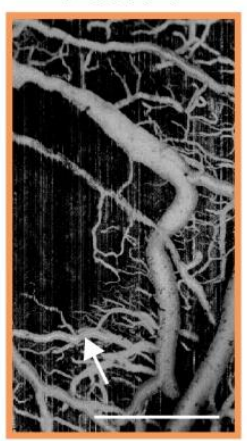

D

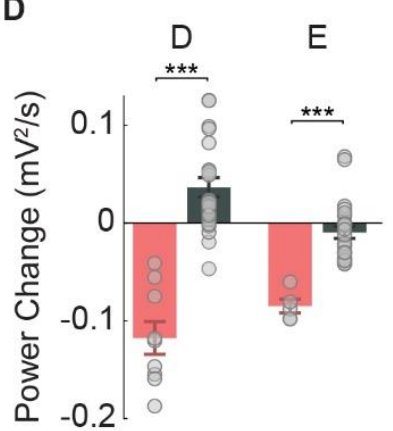

\section{E}

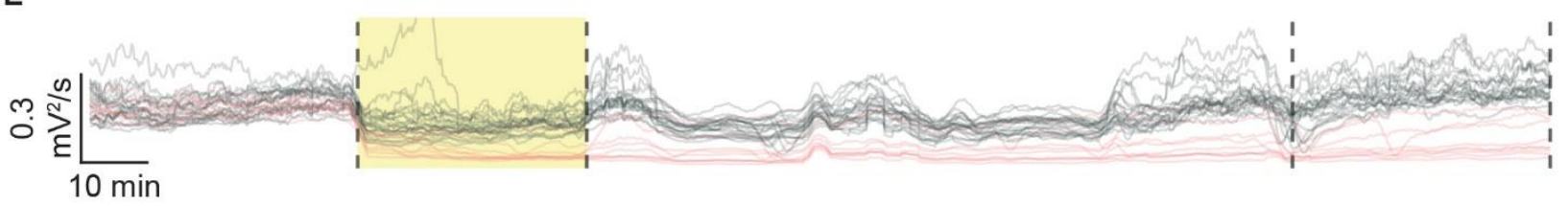

Figure 6. Large-scale electrocorticographic $(\mathrm{ECoG})$ recording of neural activity before, during,

3 and after photothrombotic lesion induction. (A), The transparency of the array enabled OCTA

$4 \quad$ imaging through the array. An example shown here where the area denoted by the green square

5 was imaged in monkey D. Scale bar is $3 \mathrm{~mm}$ in the right panel and $2 \mathrm{~mm}$ in the left panel. (B),

6 Baseline and 3 hours post-photothrombosis OCTA images demonstrating lesion induction. The

7 imaged areas shown is denoted by the orange rectangle in (A). Scale bar is $2 \mathrm{~mm}$. (C) Gamma

8 band power $(30-59 \mathrm{~Hz})$ was calculated from 30 minutes of ECoG recording before and 2.5 hours

9 post-photothrombosis for each channel across the array. The change in power was then calculated

10 for each channel. Channels with statistically significant reductions in gamma power were 
1 identified (in pink, paired left-tailed t-test, family-wise error rate $\leq 0.001$ ). Yellow circle indicates

2 the location and extent of the aperture for photothrombotic lesion induction. (D) The average

3 change in power for the lesioned group versus the non-lesioned group (error bars denote \pm standard

4 error of the mean, SEM; one-way ANOVA, monkey D: $\mathrm{p}=5.2 \mathrm{e}-9$, monkey E: $\mathrm{p}=1.0 \mathrm{e}-5)$. (E)

5 Example of neural recording (gamma power) through our ECoG array in monkey D as the lesions

6 were induced. Illumination period is shown in yellow. The traces are color-coded according to (D)

7 to show the difference between the lesioned and non-lesioned areas.

A

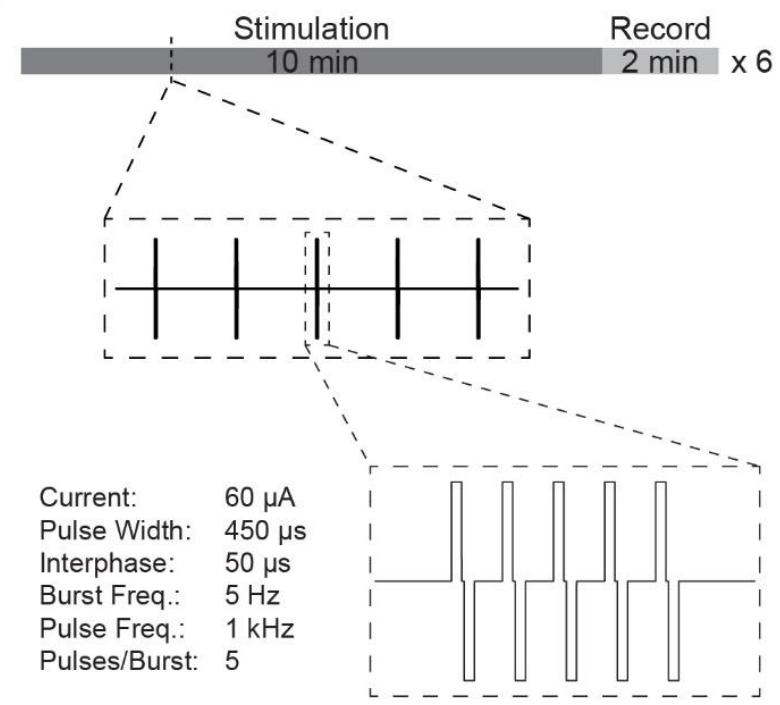

B

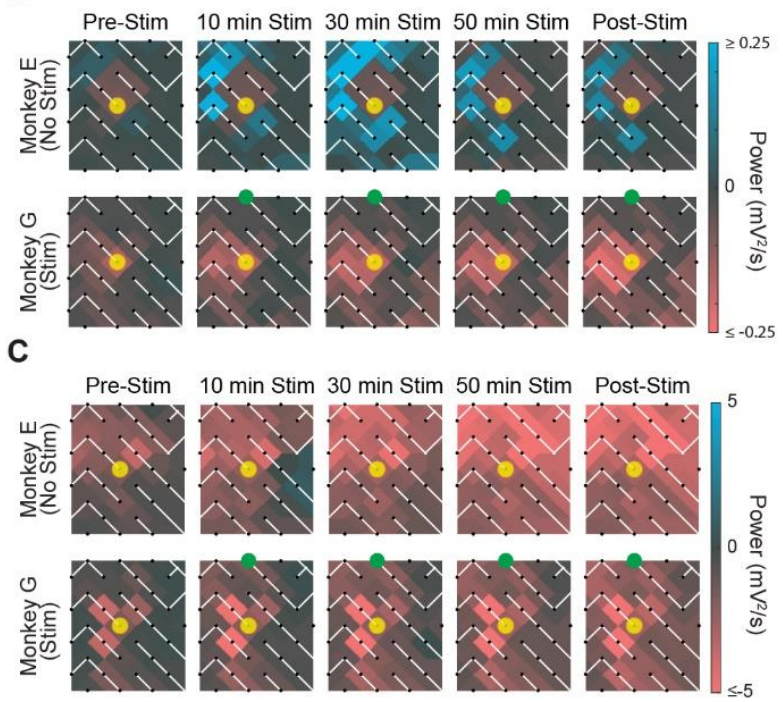

D

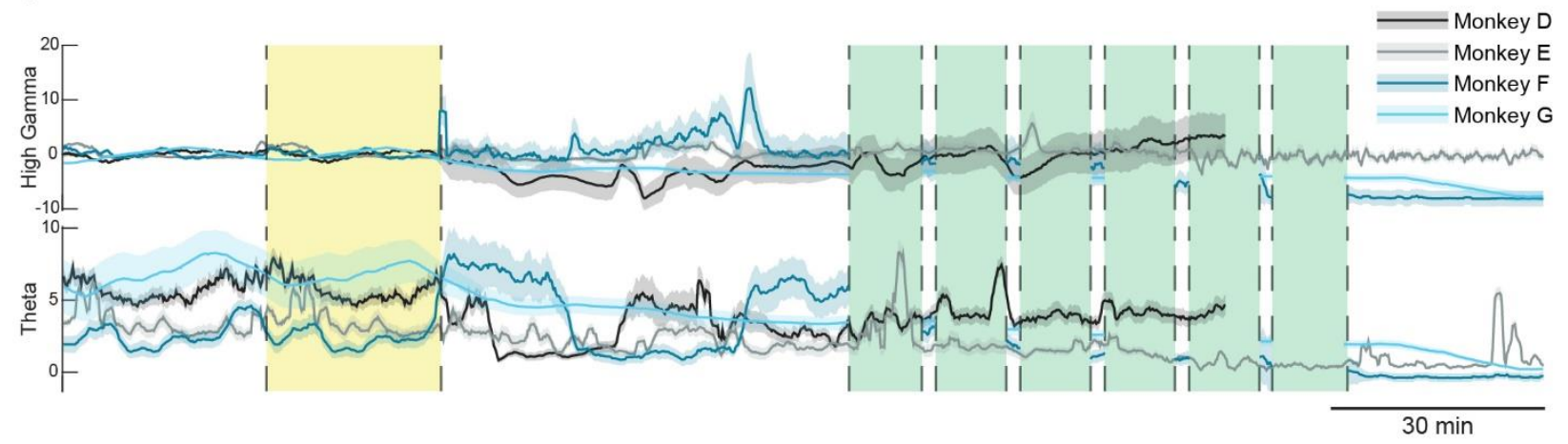

9 Figure 7. Electrical stimulation enables modulation of peri-lesional neural activity. (A)

10 Stimulation protocol used in this study about one hour after illumination period. Six 10-minute 
1 blocks of stimulation were interspaced with 2 min of recording blocks. (B,C) Heat maps of change

2 in high gamma band (B) and theta band (C) power across the ECoG array following blocks of

3 stimulation with respect to baseline for monkeys E (no stimulation) and monkey G (stimulation).

4 Yellow circle indicates the illuminated area and the stimulated channel is indicated by the green

5 node. (D) Average time course of high gamma and theta band power for monkeys D-G. The yellow

6 shaded area indicates the illumination period, while the green shaded areas indicate stimulation

7 blocks for monkeys $\mathrm{F}$ and $\mathrm{G}$. Power is normalized to baseline, and the mean power $\pm 2 \times$ SEM is

8 shown for each animal.

\section{STAR Methods}

10 Key Resources Table

\begin{tabular}{|l|l|l|}
\hline REAGENT or RESOURCE & SOURCE & IDENTIFIER \\
\hline Antibodies & Millipore-Sigma & $\begin{array}{l}\text { Cat\# MAB377; } \\
\text { RRID: AB_2298772 }\end{array}$ \\
\hline Mouse monoclonal anti-NeuN & $\begin{array}{l}\text { Invitrogen } \\
\text { ReadyProbes }\end{array}$ & $\begin{array}{l}\text { Cat\# R37114; } \\
\text { RRID:AB_2556542 }\end{array}$ \\
\hline Donkey anti-Mouse IgG (H+L), Alexa Fluor 488 & & \\
\hline & & \\
\hline & & \\
\hline Bacterial and virus strains & & \\
\hline & & \\
\hline & & \\
\hline & & \\
\hline & & \\
\hline Biological samples & & \\
\hline & & Cat\# KE- \\
\hline & & Cat\# 441244-3KG \\
\hline & Shin-Etsu & \\
\hline & & \\
\hline Chemicals, peptides, and recombinant proteins & & \\
\hline Silicone base and polymer & & \\
\hline Paraformaldehyde, prilled & & \\
\hline
\end{tabular}




\begin{tabular}{|c|c|c|}
\hline Cresyl Violet & $\begin{array}{l}\text { FD } \\
\text { Neurotechnologies, } \\
\text { Inc. }\end{array}$ & Cat\# PS102-01 \\
\hline Thionin Acetate salt, Lauth's Violet & Sigma Aldrich & Cat\# 861340-5G \\
\hline Triton-X100 & Fisher Scientific & Cat\# BP151500 \\
\hline VectaShield mounting medium & Vector Laboratories & $\mathrm{H}-1000-10$ \\
\hline Normal Donkey Serum & $\begin{array}{l}\text { Jackson } \\
\text { ImmunoResearch Lab }\end{array}$ & Cat\# 017-000-121 \\
\hline \multicolumn{3}{|l|}{ Critical commercial assays } \\
\hline & & \\
\hline & & \\
\hline \multicolumn{3}{|l|}{ Deposited data } \\
\hline Code for power analysis & This paper & $\mathrm{N} / \mathrm{A}$ \\
\hline Code for photothrombotic quantitative model & This paper & $\mathrm{N} / \mathrm{A}$ \\
\hline \multicolumn{3}{|l|}{ Experimental models: Cell lines } \\
\hline & & \\
\hline & & \\
\hline & & \\
\hline \multicolumn{3}{|l|}{ Experimental models: Organisms/strains } \\
\hline Macaca mulatta/nemestrina & $\begin{array}{l}\text { Washington National } \\
\text { Primate Research } \\
\text { Center }\end{array}$ & $\mathrm{N} / \mathrm{A}$ \\
\hline & & \\
\hline & & \\
\hline & & \\
\hline \multicolumn{3}{|l|}{ Oligonucleotides } \\
\hline & & \\
\hline & & \\
\hline & & \\
\hline \multicolumn{3}{|l|}{ Recombinant DNA } \\
\hline & & \\
\hline & & \\
\hline & & \\
\hline & & \\
\hline Software and algorithms & & \\
\hline
\end{tabular}




\begin{tabular}{|l|l|l|}
\hline MATLAB R2020a & MathWorks & $\begin{array}{l}\text { RRID:SCR_001622, } \\
\text { http://www.mathwork } \\
\text { s.com/products/matl } \\
\text { ab// }\end{array}$ \\
\hline Hugin-2019.2.0 & Hugin Software & $\begin{array}{l}\text { http://www.mathwork } \\
\text { s.com/products/matl } \\
\text { ab/ }\end{array}$ \\
\hline Monte Carlo eXtreme (MCX) & Fand and Boas, 2009 & $\begin{array}{l}\text { RRID:SCR_007001, } \\
\text { http://mcx.sourceforg } \\
\text { e.net/ }\end{array}$ \\
\hline Adobe Photoshop & Adobe & $\begin{array}{l}\text { RRID:SCR_014199, } \\
\text { https://www.adobe.c } \\
\text { om/products/photosh } \\
\text { op.html }\end{array}$ \\
\hline Other & & \\
\hline & & \\
\hline & & \\
\hline & & \\
\hline
\end{tabular}

2 Resource Availability

3 Lead Contact

4 Further information and requests for resources and reagents should be directed to and will be

5 fulfilled by the lead contact, Azadeh Yazdan-Shahmorad (azadehy@uw.edu).

$6 \quad$ Materials Availability

7 This study did not generate new unique reagents.

8 Data and Code Availability

9 The data supporting the findings of this study and code used to generate the figures and develop

10 the predictive quantitative model are available from the lead contact upon request.

\section{Experimental Model and Subject Details}

12 All animal procedures were approved by the University of Washington Institutional Animal Care

13 and Use Committee. 


\section{$1 \quad$ Method Details}

2 Surgical Procedure and Photothrombotic Technique

3 Three adult Macaca mulatta (monkey A, $18.45 \mathrm{~kg}, 14$ years, male; monkey B, $10.75 \mathrm{~kg}$, 16 years,

4 male; monkey C, $10.3 \mathrm{~kg}, 16$ years, female) were anesthetized with isoflurane and placed in a

5 stereotaxic frame. Throughout the procedure we used standard aseptic technique, and the animals'

6 body temperature, heart rate, electrocardiographic responses, oxygen saturation, and $\mathrm{CO}_{2}$ end-tidal

7 pressure were monitored. A sagittal or coronal incision was made to expose underlying soft tissue

8 in the right hemisphere of monkey A and bilaterally in monkeys B and C. After removing the soft

9 tissue to reveal the skull, a $25 \mathrm{~mm}$ diameter craniotomy targeting the sensorimotor cortex was

10 made based on the macaque brain atlas (Paxinos et al., 2009) with a center $17.5 \mathrm{~mm}$ from the

11 midline and $11.55 \mathrm{~mm}$ from the interaural line using a trephine in the right hemisphere of monkey

12 A and bilaterally in monkeys B and C. To allow for optical access to the cortical surface, we

13 performed a durotomy to excise the opaque native dura and replaced it with a transparent silicone

14 artificial dura (10:1 Shin-Etsu KE1300-T and CAT-1300) of $0.5 \mathrm{~mm}$ thickness and $25 \mathrm{~mm}$

15 diameter. We acquired baseline OCTA images of the exposed cortical surface through the artificial

16 dura as described later in a later section. Light exposure was limited across the cortical surface by

17 placing on the artificial dura an opaque silicone mask with circular apertures with diameters of

$180.5,1.0$, and $2.0 \mathrm{~mm}$ distributed across the mask (Figure 1A). We then intravenously injected 20

$19 \mathrm{mg} / \mathrm{kg}$ of Rose Bengal solution $(40 \mathrm{mg} / \mathrm{mL}$ concentration) through the saphenous vein over the

20 course of 5 minutes and began illumination through the mask with a cold white light source for 30

21 minutes (Figure 1B). To prevent further light exposure following illumination, the cranial window

22 was optically shielded until OCTA images were acquired 3 hours post-illumination. Following 
1 post-illumination OCTA imaging, we perfused the animal with $6 \mathrm{~L}$ of $4 \%$ paraformaldehyde then

2 extracted and immersed the brain in $4 \%$ paraformaldehyde solution for $24-48$ hours at $4{ }^{\circ} \mathrm{C}$.

3 Electrocorticographic Recording, Stimulation, and Power Analysis

\section{$4 \quad$ Electrocorticographic Recording}

5 Four adult Macaca nemestrina (monkey D, $12.8 \mathrm{~kg}, 14$ years, female; monkey E, $13.10 \mathrm{~kg}, 14$

6 years, female; monkey F, $13.8 \mathrm{~kg}, 14$ years, female; monkey G, $14.6 \mathrm{~kg}, 7$ years, male) underwent

7 a surgical procedure similar to that of monkeys A, B, and C. Briefly, bilateral craniotomies and

8 durotomies were performed targeting the sensorimotor cortex following sedation and stereotaxic

9 positioning. After baseline OCTA images were acquired through the transparent silicone artificial

10 dura (monkeys D-E), the artificial dura was removed and replaced with a semi-transparent ECoG

11 array of 32 electrodes (Figure 4A). To correlate electrode location with lesion location, OCTA

12 images were acquired prior to photothrombosis through the array for monkey D (Figure 4A). We

13 placed an opaque mask similar to that of monkeys $\mathrm{A}$ through $\mathrm{C}$ but with a single aperture located

14 in the center with a diameter of $1.5 \mathrm{~mm}$ on the ipsilesional hemisphere (monkeys D, F, and G: left

15 hemisphere; monkey E: right hemisphere). In the contralesional hemisphere we placed a mask

16 without any apertures. A skull screw located anterior and medial to the ipsilesional cranial window

17 was used as a ground for ECoG recordings. Prior to Rose Bengal infusion and illumination as

18 described previously, baseline ECoG recordings were collected bilaterally for 30 minutes. We also

19 recorded LFPs during illumination and for 3 hours following illumination. We then acquired

20 OCTA images in both hemispheres (monkeys D-E), perfused the animals, and stored the extracted

21 brain as described previously. 
2 One hour following illumination, we stimulated perilesionally through one channel for a duration

3 of about one hour in monkeys $\mathrm{F}$ and $\mathrm{G}$. Stimulation $(60 \mu \mathrm{A}, 5$ biphasic pulses per burst at $1 \mathrm{kHz}$,

$4450 \mu$ s pulse width, $50 \mu$ s interphase interval, $5 \mathrm{~Hz}$ burst frequency) occurred in six ten-minute

5 blocks interspaced with 2-minute recording blocks. Post-stimulation recording was then continued

6 for another 30-60 minutes.

7 Power Analysis

8 LFPs were recorded at a rate of $30 \mathrm{kHz}$ and downsampled to $1 \mathrm{kHz}$ in MATLAB for power

9 analysis. Signals were notch filtered at $60,120,180$, and $240 \mathrm{~Hz}$, and were bandpass filtered to

10 isolate theta $(4-7 \mathrm{~Hz})$, gamma $(30-59 \mathrm{~Hz})$, and high gamma $(60-150 \mathrm{~Hz})$ bands. After filtering,

11 artifacts were removed from the signal by normalizing the discrete time series of each signal, and

12 samples with an amplitude exceeding 25 standard deviations were identified and excluded from

13 analysis. Similarly, channels with power spectral densities that did not exhibit the expected $1 / \mathrm{f}^{\alpha}$

14 curve were also excluded from analysis. Thus, one channel was excluded from analysis in monkey

15 D, three channels were excluded from analysis for monkey E, and 11 channels were excluded from

16 analysis for monkey F. No channels were excluded for monkey G. The signal power for each

17 frequency band was calculated for each electrode over the course of the baseline and the final 30

18 minutes of post-illumination recording periods. Signal power was calculated by squaring the

19 filtered signal and dividing by the elapsed time. Change in signal power was calculated by

20 subtracting mean baseline power from the mean post-illumination power. To isolate channels with

21 decreasing power, we performed a left-tailed paired t-test between the 30 minutes of baseline

22 power and the final 30 minutes of post-illumination power calculated at $60 \mathrm{~s}$ intervals (family-wise

23 error rate $\leq 0.001$ to account for multiple comparisons). Thus, we categorized channels in a 
1 lesioned group (decreasing power), and a non-lesioned group (failed to show a decrease in power).

2 A one-way ANOVA was used to compare average change in power of channels across the two

3 groups at a significance level of 0.05 . The time-varying signal power across all recording periods

4 was calculated in $10 \mathrm{~s}$ intervals and smoothed (smoothing factor of 0.4-0.5). To observe the effects

5 of electrical stimulation over time, the power time-course for each individual channel was

6 normalized to baseline mean and standard deviation.

7 Histology and Immunohistochemistry

8 After 24-48 hours of post-fixation in 4\% paraformaldehyde brains of all animals were dissected

9 into a single $25 \mathrm{~mm}$ thick coronal block containing the region of interest using a custom matrix

10 and stored in $30 \%$ sucrose in PBS solution at $4{ }^{\circ} \mathrm{C}$ for a minimum of one week. The block was

11 then frozen and sectioned into $50 \mu \mathrm{m}$ coronal sections using a cryostat (Microm, Thermo Fisher)

12 or a sliding microtome (Leica). Cut sections were stored in PBS solution with $0.02 \%$ sodium azide

13 at $4{ }^{\circ} \mathrm{C}$. To evaluate the extent of ischemic damage to the neuronal population we first performed

14 Nissl staining on mounted serial coronal sections with a rostrocaudal separation of approximately

150.45 mm using Cresyl Violet (monkeys A and B) or Thionin acetate (monkeys C, D, and E). Due

16 to inadequate storage conditions resulting in loss of tissue, not all putative lesions were confirmed

17 histologically for monkey A. Therefore, data from monkey A was excluded from the rest of our

18 analysis. To additionally confirm the neuronal cell loss in the illuminated regions, we

19 immunostained adjacent sections for a selective neuronal marker NeuN. Sections were further

20 trimmed to include the mediodorsal part of the hemisphere with putative lesion sites; these were

21 first incubated in $50 \%$ alcohol for 20 min to permeabilize, then rinsed in PBS and incubated in

$2210 \%$ normal donkey serum in PBS containing $0.1 \%$ triton-X100 at $4{ }^{\circ} \mathrm{C}$ for $1-2$ hours, and then

23 incubated with a mouse anti-NeuN primary antibody (1:500, Millipore Sigma; MAB377, RRID: 
1 AB_2298772) at $4{ }^{\circ} \mathrm{C}$ for 48 hours. Sections were then rinsed in PBS, incubated in $2 \%$ normal

2 donkey serum followed by the secondary donkey anti-mouse antibody conjugated to AlexaFluor

3488 (1:300, Millipore) for 4-6 hours at room temperature. Sections were rinsed and mounted using

$4 \quad$ VectaShield mounting media (Vector) containing DAPI and imaged using Nikon 6D widefield

5 automated microscope system (Nikon imaging Center at UCSF).

6 Lesion Reconstruction and Size Measurement

7 All Nissl-stained slices for monkeys B through E were imaged and co-registered in MATLAB

8 (MATLAB R2020a, MathWorks) without resizing. The registered images were then edited in

9 Adobe Photoshop to enhance boundaries between infarct and non-infarcted regions, after which

10 each slice was smoothed with an edge-preserving filter, binarized, and underwent edge-detection

11 to identify slice boundaries with and without the infarcted regions in MATLAB (Figure 2D,E).

12 Lesion boundaries were obtained by subtracting boundaries with the lesions from boundaries that

13 excluded the lesions. These boundaries were then visualized as surfaces in three-dimensional space

14 with linear interpolation of the approximately $0.45 \mathrm{~mm}$ rostrocaudal separation between slices.

15 The average diameter and maximum depth from representative coronal slices of each lesion were

16 then calculated based on image resolution.

17 Optical Coherence Tomography Angiography Imaging

18 For in vivo validation of disrupted blood flow in cortical vasculature, we acquired OCTA images

19 for monkeys B through E. OCTA was acquired from a custom-built prototype OCT system, using

20 a $200 \mathrm{kHz}$ vertical cavity surface-emitting swept source (SL1310V1-10048, Thorlabs Inc.,

21 Newton, NJ) with a central wavelength of $1310 \mathrm{~nm}$, and a sweeping bandwidth of $100 \mathrm{~nm}$. The

22 prototype was similar to the device reported in a previous publication (Deegan et al., 2018b; Xu et

23 al., 2017). 
1 OCTA was performed in repeated raster-scan. The scanning beam was directed into the cortical

2 tissue through a transparent silicone artificial dura, prior to photothrombosis and 3 hours post-

3 illumination with a lateral FOV of approximately $9 \mathrm{~mm} \times 9 \mathrm{~mm}$. At each position on the raster

4 scan, one full A-scan approximately $8 \mathrm{~mm}$ deep (1408-sampling-pixel) was acquired. Each B-

5 frame contained 1000 A-line scans and was repeated 8 times, then moved to the next B-frame.

6 This process was repeated to form a volume of 1408 x 1000 A-lines x 8 repeat x 1000 B-frames.

7 The optimal resolution in the axial direction (into the tissue) was $5.5 \mu \mathrm{m}$ and the lateral resolution

8 was $24 \mu \mathrm{m}$. The volume scan was repeated at different regions within the cranial window, then

9 processed to form a 3D OCTA data set. For visualization, the 3D OCTA was color-coded with the

10 depth associated with each vessel into a 2D-en-face (top-down) projection.

11 The 2D-en-face projections at different regions were then stitched together to form a larger FOV

12 as in Figure 3 using the Hugin software (Hugin-2019.2.0). Stitched OCTA images were then

13 aligned with images of the cortical surface via control point registration in MATLAB. To estimate

14 the diameters of the lesions, images were first smoothed with a Gaussian filter and binarized.

15 Lesion boundaries of each detected lesion were fitted with ellipses and the major and minor axis

16 lengths were recorded. The major axis lengths were then defined as OCTA-measured lesion

17 diameters. Illuminated regions outside the FOV excluded from analysis.

18 Light Intensity Measurements

19 Due to the uncollimated nature of the illuminating light beam, the power density of the light

20 through each aperture was assumed to differ based on the location and diameter of each aperture.

21 We measured the light intensity $(535 \mathrm{~nm})$ through each aperture (10-20 times) and through the

22 mask without any apertures using a photodetector (Thorlabs PM100D Power Meter and S121C

23 Sensor). 


\section{$1 \quad$ Lesion Size and Illumination Parameter Analysis}

2 The relationship between OCTA-measured lesion diameter and histologically measured lesion

3 diameter was assessed by fitting an ordinary least squares linear regression in MATLAB (fitlm

4 function). Similarly, the relationships between OCTA-measured diameter and histological lesion

5 depth, as well as histological depth and diameter were analyzed by ordinary least squares

6 regression. We also modeled the combined effects of both aperture diameter and the log-

7 transformed light intensity as predictor variables of each lesion size metric (OCTA-measured

8 diameter, histological diameter, and histological depth) separately in MATLAB by fitting a

9 multivariate ordinary least squares linear regression (fitlm function). To better visualize the

10 multivariate regressions, we plotted partial regressions, in which the adjusted variables represent

11 the mean of the plotted variable plus the residual of the variable fit with the excluded predictor

12 variable (Figure 4G-L).

\section{Light Simulation}

14 Our computational model of light penetration and subsequent lesion in the brain consisted of a

15 Monte Carlo simulation of photons propagating through brain tissue, extraction of a light intensity

16 contour, and scaling of the contour. A virtual volume was constructed which mimicked the

17 experimental setup and had the relevant optical properties of brain tissue. Photons were virtually

18 propagated through this volume to obtain a probability distribution of photon fluence, or light

19 energy passing through a given area. Optical parameters were informed by published literature

20 regarding primate cortical optical properties and then refined to maximally match our experimental

21 results obtained through histology. The Monte Carlo simulations were designed using the Monte

22 Carlo eXtreme (MCX) software (Fang and Boas, 2009) and run on discrete graphics cards (Nvidia

23 GTX 1080Ti and Nvidia GTX 2060). 
1 The virtual volume consisted of a light source and mask, an artificial dura, and gray and white

2 matter (Figure 5A). The entire volume was $8 \mathrm{~mm}$ x $8 \mathrm{~mm}$ x $5.6 \mathrm{~mm}$ (width $\mathrm{x}$ width $\mathrm{x}$ height), with

3 voxel resolution of $0.01 \mathrm{~mm}^{3}$. We specified the light source as a disk parallel to the surface of the

4 brain with collimated light exiting the side of the disk facing the brain, and then passing through a

5 thin highly scattering disk which uncollimated the light. We set the diameters of both disks to be

6 equal to the aperture diameter of the mask of the corresponding in vivo experiment. We specified

7 the volume peripheral to the disks as a highly absorbing and highly scattering medium to mimic

8 the opacity of the non-aperture part of the mask (Supplementary Table 2). The diameter used in

9 our simulations were $0.5 \mathrm{~mm}, 1 \mathrm{~mm}, 1.5 \mathrm{~mm}$, and $2 \mathrm{~mm}$, corresponding with the apertures tested

10 in our in vivo experiments. After specifying the volumes, we ran simulations of 1 million photons

11 through the volume. The simulations yielded voxel-wise fluence values, or values of radiant energy

12 received per unit area. The fluence results were imported into MATLAB and analyzed with custom

13 code.

14 Research on the optical properties of gray matter is often contradictory, and values of index of

15 refraction, anisotropy coefficient, and absorption and scattering coefficients vary by orders of

16 magnitude in published research. We defined a grid search boundary of optical properties by using

17 the values reported in previous literature (Gottschalk, 1992; Yaroslavsky et al., 2002) as upper and

18 lower bounds of the properties. As the absorption spectrum for Rose Bengal peaks at $559 \mathrm{~nm}$, we

19 used the optical properties reported for this wavelength by interpolating from nearest reported

20 wavelengths. The range of the grid was $[0.03,0.76] \mathrm{mm}^{-1}$ for gray matter absorption, $[9.9,53.6]$

$21 \mathrm{~mm}^{-1}$ for gray matter scattering, $[0.09,0.36] \mathrm{mm}^{-1}$ for white matter absorption, and $[41.9,78.4]$

$22 \mathrm{~mm}^{-1}$ for white matter scattering. For anisotropy coefficients the two publications reported similar

23 values, therefore we averaged the values between the publications to obtain an anisotropy 
1 coefficient of 0.92 for gray matter and 0.8 for white matter. The refractive index was set as 1.36

2 for gray matter and 1.38 for white matter ${ }^{54}$. From the optical property ranges we constructed a $5 \mathrm{x}$

$35 \times 4 \times 4$ size grid with 5 values for the gray matter absorption and scattering coefficients and 4

4 values for the white matter absorption and scattering coefficients. For each of the 400 combinations

5 of parameters, four simulations were run, corresponding with the four aperture sizes tested in our

6 experiments. Each simulation yielded a volumetric fluence distribution. We further obtained

7 representative $2 \mathrm{D}$ central slices to compare with histologically obtained lesions.

8 For each photon intensity distribution corresponding to an optical property combination, we

9 identified the light intensity threshold which yielded contours most closely matching those

10 obtained from central slices of lesions obtained from histology. By treating the maximum depth

11 and average width of an individual contour as the prediction of maximum depth and average width

12 of the corresponding lesion, we quantified the predictive power of the simulation. Denote by $S S R_{d}$

13 the residual sum of squares of prediction of maximum depth of a lesion, $S S T_{d}$ the total sum of

14 squares of maximum depth of a lesion, $S S R_{w}$ the residual sum of squares of prediction of average

15 width of a lesion, and $S S T_{w}$ the total sum of squares of average width of a lesion. The objective

16 function used to find the best light intensity threshold was:

17

$$
\min _{\text {thresh }}\left(\frac{S S R_{d}^{2}}{S S T_{d}^{2}}+\frac{S S R_{w}{ }^{2}}{S S T_{w}{ }^{2}}\right)
$$

18 This is equivalent to maximizing the (squared or unsquared) Euclidean norm of the r-squared 19 values of depth and width predictions less 1 . Denote by $r_{d}^{2}$ the r-squared value of the simulation- 
1 based prediction of maximum depth of a lesion, and $r_{w}^{2}$ the r-squared value of the simulation-based

2 prediction of average width of a lesion. Then the equation above can be rewritten as:

$$
\max _{\text {thresh }}\left(\left(r_{d}^{2}-1\right)^{2}+\left(r_{w}^{2}-1\right)^{2}\right)
$$

3 We solved this objective function individually for each optical property combination using

4 Bayesian optimization. We then identified the simulation yielding the minimum value of this

5 objective function as that most closely matching our histology data and used the results of that

6 simulation for all later parts of the analysis. The optical properties of this best simulation were

7 gray matter absorption coefficient of $0.395 \mathrm{~mm}^{-1}$, gray matter scattering coefficient of $53.6 \mathrm{~mm}^{-1}$,

8 white matter absorption coefficient of $0.09 \mathrm{~mm}^{-1}$, white matter scattering coefficient of 54.066

$9 \mathrm{~mm}^{-1}$, and a light intensity threshold for lesion induction of $19.9 \mu \mathrm{W} / \mathrm{mm}^{2}$.

10 We then identified a transformation to accurately scale the profiles of the light simulation contours

11 to that of the lesions obtained from histology (Figure 5C,D). We opted to do this by independently

12 scaling the width and depth of the contours. Our first approach was to fit a univariate linear

13 regression from the simulation depths and widths to the lesion depths and widths, respectively. In

14 this linear regression we did not allow an intercept term. Based on the results of our linear

15 regressions, we further evaluated the need for non-linear transformations. Once these scaling

16 factors were set, we treated them as the final stage of our modeling platform, whereby light

17 intensity contours from the Monte Carlo would be stretched and shrunk accordingly to transform

18 from the light profile to the lesion profile. 


\section{Supplemental Information Titles and Legends}
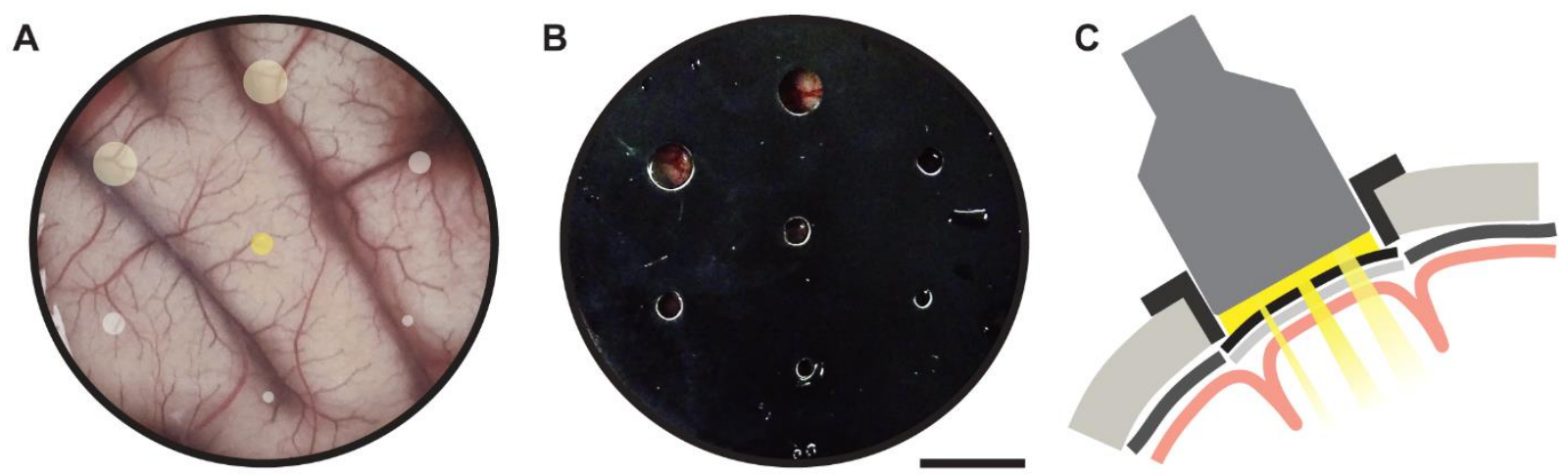

3 Supplementary Figure 1. Schematic of illumination using a multi-apertured mask used for

4 monkeys A, B, and C. (A) A cranial window through an artificial dura with projected areas of

5 illumination shown in yellow. (B) Multi-apertured mask is placed on top of the artificial dura. (C)

6 Coronal schematic of illumination through the apertured mask. This setup enables testing different

7 illumination intensities and aperture diameters.
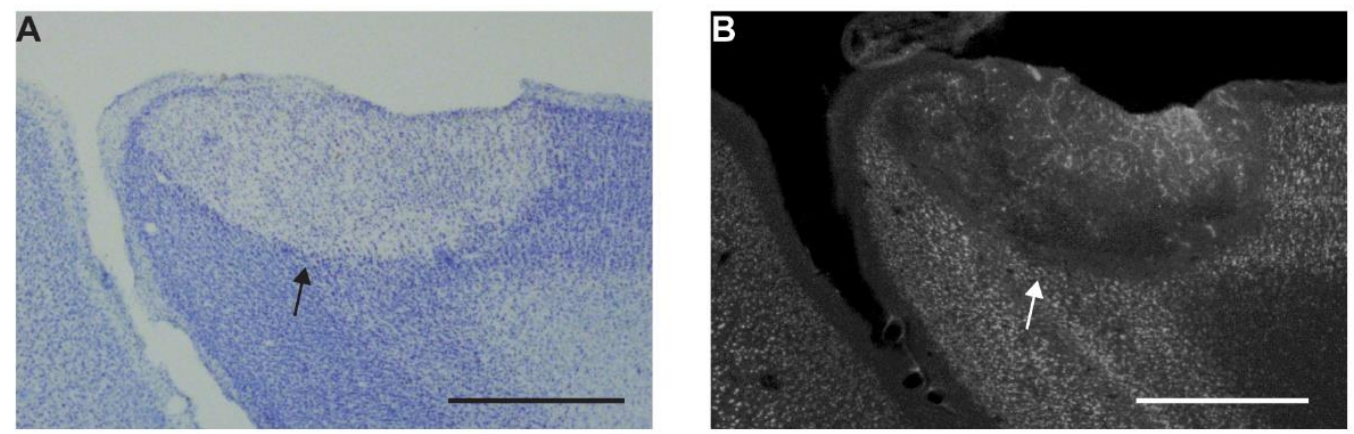

9 Supplementary Figure 2. Validation of neuronal cell loss with NeuN staining. (A) Nissl staining

10 demonstrating cell loss indicated by the black arrow in a representative coronal slice from monkey

11 C. (B) NeuN staining of an adjacent slice demonstrating neuronal cell loss in the same lesion

12 indicated by the white arrow. Scale bars are $1 \mathrm{~mm}$. 


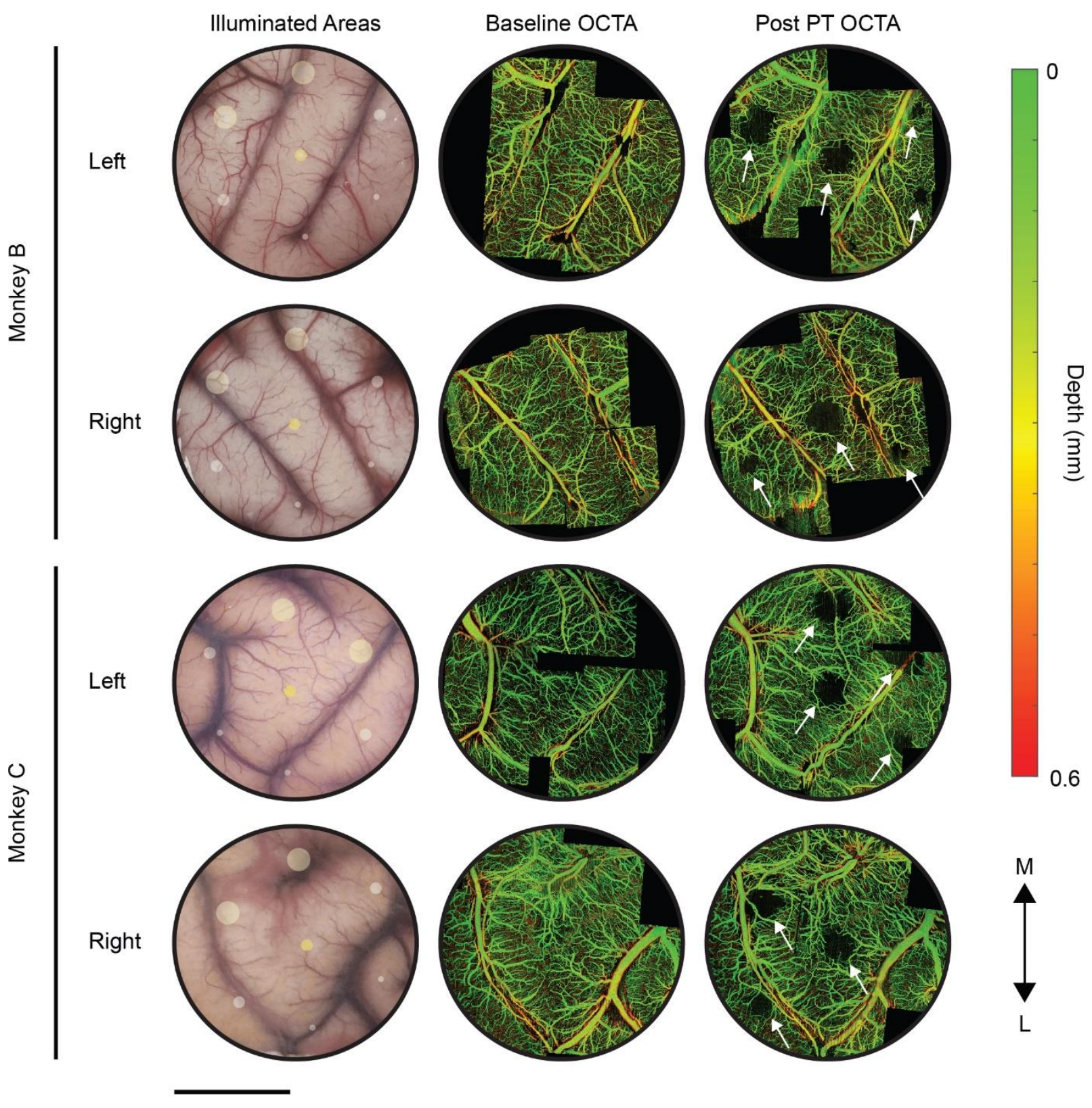

2 Supplementary Figure 3. Optical coherence tomography angiography (OCTA) imaging was used

3 to validate lesion induction in vivo. Stitched baseline and post-photothrombosis OCTA images are

4 shown for both hemispheres of monkeys B and C, with vasculature color indicating cortical depth.

5 In the left column, yellow circles indicate the illuminated regions for photothrombosis, with the

6 diameters corresponding with the aperture diameters. Shading of the yellow circles in the left

7 column indicate relative intensity of illumination. Baseline OCTA images are shown in the middle 
1 column. In the right column, dark regions indicated by white arrows in OCTA images taken 3

2 hours after photothrombosis indicate successful lesion induction due to the occlusion of the

3 illuminated microvasculature. Scale bar is $1 \mathrm{~cm}$.

A

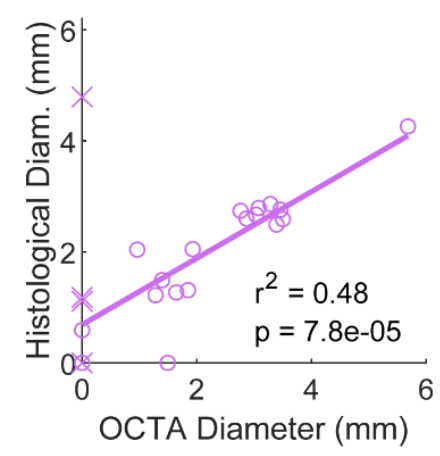

C

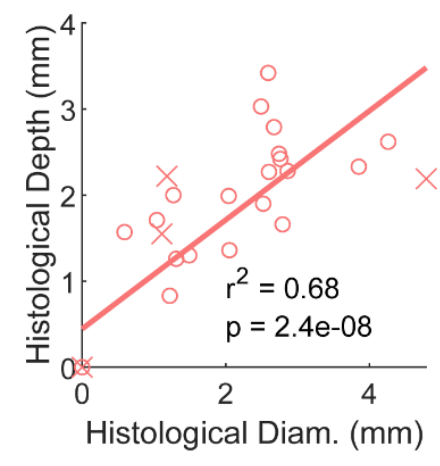

B

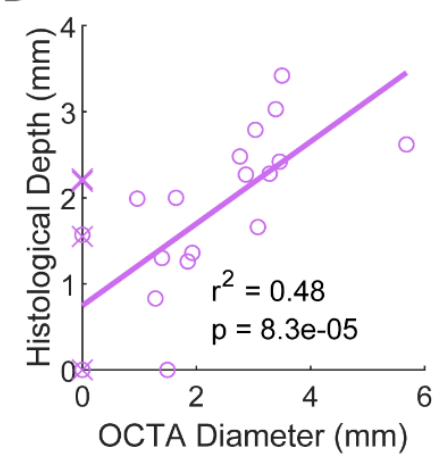

D

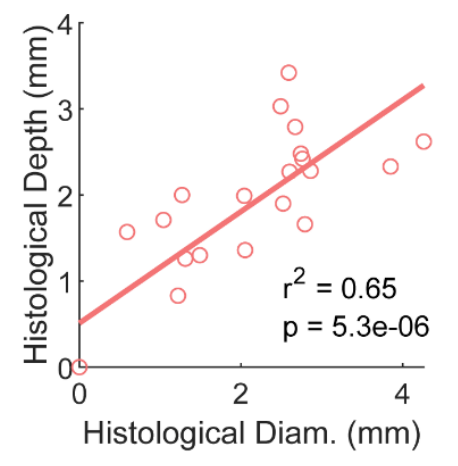

5 Supplementary Figure 4. OCTA predictions of histologically measured lesion size with large vessel values included. (A) Correlation between OCTA- and histologically measured lesion

7 diameters (0.49 r-squared, $\mathrm{p}=7.8 \mathrm{e}-5)$. (B) Correlation between OCTA-measured lesion diameters

8 and histologically measured lesion depths (0.48 r-squared, $\mathrm{p}=8.3 \mathrm{e}-5)$. (C,D) Correlation between

9 histologically measured lesion diameters and depths with large vessel values included (C, 0.68 r-

10 squared, $\mathrm{p}=2.4 \mathrm{e}-8)$, and excluded ( $\mathrm{D}, 0.65 \mathrm{r}$-squared, $\mathrm{p}=5.3 \mathrm{e}-6)$. 

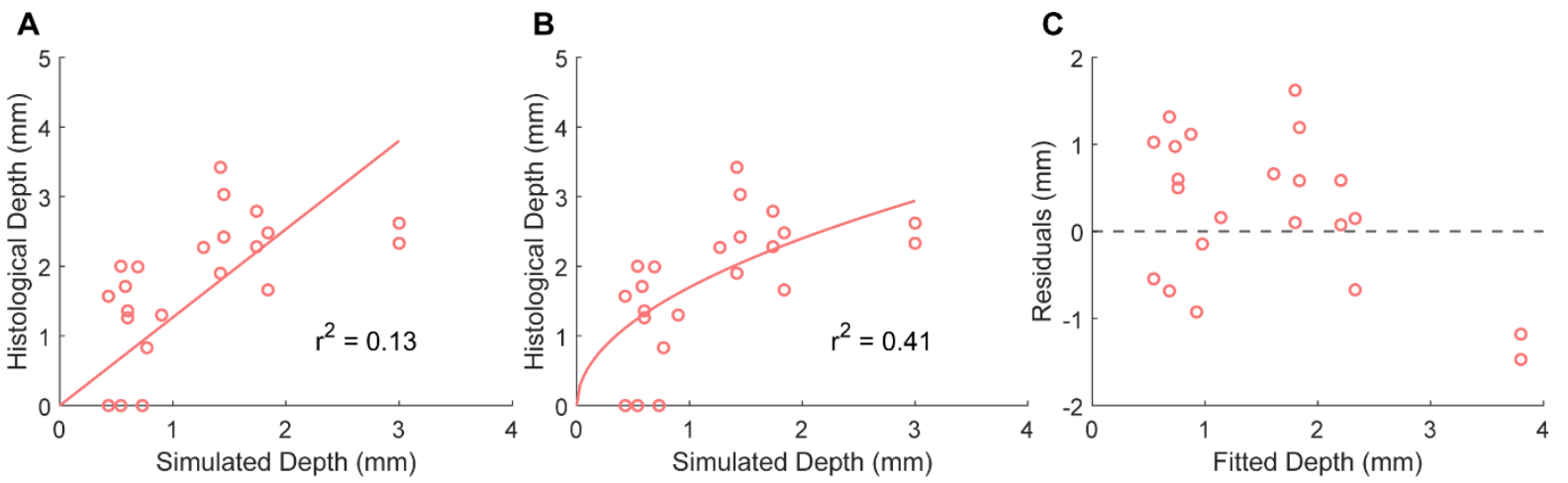

2 Supplementary Figure 5. Transformation of simulated depths in second stage of modeling

3 photothrombotic lesions. (A) Histological depths vs linearly transformed simulated depths. (B)

4 Histological depths vs square root square root transformed simulated depths. (C) Residuals of

5 histological depths vs linearly transformed simulated depths plotted against fitted values from (A).

Intercept $(\mathrm{mm})$ $\log ($ Intensity $(\mathrm{mW}))$

Aperture Diameter (mm)

\begin{tabular}{|c|c|}
\hline \multicolumn{2}{|c|}{ OCTA Diameter } \\
\hline Estimate & p Value \\
\hline 2.0381 & \\
\hline $\begin{array}{l}0.57968 \\
0.90238\end{array}$ & $\begin{array}{l}7.7597 \mathrm{e}-06 \\
0.0066161\end{array}$ \\
\hline
\end{tabular}

\begin{tabular}{|c|c|}
\hline \multicolumn{2}{|c|}{ Histological Diameter } \\
\hline Estimate & p Value \\
\hline 1.9002 & \\
\hline $\begin{array}{l}0.46653 \\
0.55467\end{array}$ & $\begin{array}{l}2.23 e-06 \\
0.036591\end{array}$ \\
\hline
\end{tabular}

\begin{tabular}{|c|c|}
\hline Hist & I Depth \\
\hline stimate & $\mathrm{p}$ Value \\
\hline & \\
\hline & \\
\hline .71 & 0.03753 \\
\hline
\end{tabular}

Supplementary Table 1. Table of the estimated coefficients and corresponding p-values for multivariate prediction of each lesion size metric.

\begin{tabular}{|c|c|c|c|c|c|}
\hline & $\underline{\mu_{a}\left(m^{-1}\right)}$ & $\mu_{\mathrm{s}}\left(\mathrm{mm}^{-1}\right)$ & $\begin{array}{l}\text { g } \\
\text { (anisotropy) }\end{array}$ & $\begin{array}{l}\mathrm{n} \text { (index of } \\
\text { refraction) }\end{array}$ & $\begin{array}{l}\text { Thickness } \\
(\mathrm{mm})\end{array}$ \\
\hline Scattering disk & 0 & 100 & 0.86 & 1.44 & 0.1 \\
\hline Mask (opaque) & 1000 & 1000 & 1 & 1.3 & 0.1 \\
\hline Artificial dura & 0.01 & 0.01 & 0.86 & 1.43 & 0.5 \\
\hline Gray matter & 0.395 & 53.6 & 0.91 & 1.36 & 2.5 \\
\hline White matter & 0.09 & 54.066 & 0.8 & 1.38 & 2.5 \\
\hline
\end{tabular}

10 Supplementary Table 2. Table of optical properties and thicknesses of virtual media used in the 11 Monte Carlo simulation. 
Baran, U., and Wang, R.K. (2016). Review of optical coherence tomography based angiography in neuroscience. Neurophotonics 3, 010902.

Borich, M.R., Brodie, S.M., Gray, W.A., Ionta, S., and Boyd, L.A. (2015). Understanding the role of the primary somatosensory cortex: Opportunities for rehabilitation. Neuropsychologia 79, 246-255.

Bouguer, P. (1729). Essai d'optique sur la gradation de la lumière (1729) (Kessinger Publihsing, 8 2009).

9 Brinkman, J., Colebatch, J.G., Porter, R., and York, D.H. (1985). Responses of Precentral Cells

10 During Cooling of Post-Central Cortex in Conscious Monkeys.

11 Chin-Hao Chen, R., Atry, F., Richner, T., Brodnick, S., Pisaniello, J., Ness, J., Suminski, A.J.,

12 Williams, J., and Pashaie, R. (2020). A system identification analysis of optogenetically evoked

13 electrocorticography and cerebral blood flow responses. J. Neural Eng. 17, 056049.

14 Dai, P.M., Huang, H., Zhang, L., He, J., Zhao, X.D., Yang, F.H., Zhao, N., Yang, J.Z., Ge, L.J., 15 Lin, Y., et al. (2017). A pilot study on transient ischemic stroke induced with endothelin-1 in the 16 rhesus monkeys. Sci. Rep. 7, 1-12.

17 Deegan, A.J., and Wang, R.K. (2019). Microvascular imaging of the skin. Phys. Med. Biol. 64.

18 Deegan, A.J., Wang, W., Men, S., Li, Y., Song, S., Xu, J., and Wang, R.K. (2018a). Optical coherence tomography angiography monitors human cutaneous wound healing over time. Quant. Imaging Med. Surg. 8, 135-150.

Deegan, A.J., Talebi-Liasi, F., Song, S., Li, Y., Xu, J., Men, S., Shinohara, M.M., Flowers, M.E., Lee, S.J., and Wang, R.K. (2018b). Optical coherence tomography angiography of normal skin and inflammatory dermatologic conditions. Lasers Surg. Med. 50, 183-193.

24 Fang, Q., and Boas, D.A. (2009). Monte Carlo Simulation of Photon Migration in 3D Turbid 25 Media Accelerated by Graphics Processing Units. Opt. Express 17, 20178.

26 Ferrier, D. (1876). The Functions of the Brain. J. Ment. Sci.

27 Le Friec, A., Desmoulin, F., Demain, B., Davoust, C., Robert, L., Duval, T., Rémy, F., Cirillo,

28 C., and Loubinoux, I. (2020). A Reproducible New Model of Focal Ischemic Injury in the

29 Marmoset Monkey: MRI and Behavioural Follow-Up. Transl. Stroke Res. 1-14.

30 Friel, K.M., Barbay, S., Frost, S.B., Plautz, E.J., Stowe, A.M., Dancause, N., Zoubina, E. V., and 31 Nudo, R.J. (2007). Effects of a rostral motor cortex lesion on primary motor cortex hand 32 representation topography in primates. Neurorehabil. Neural Repair 21, 51-61.

33 Gerlai, R., Thibodeaux, H., Palmer, J.T., Van Lookeren Campagne, M., and Van Bruggen, N. 34 (2000). Transient focal cerebral ischemia induces sensorimotor deficits in mice. Behav. Brain 35 Res. 108, 63-71.

36 Gottschalk, W. (1992). Ein Messverfahren zur Bestimmung der optischen Parameter 37 biologischer Gewebe in vitro. 
Gould, I.G., Tsai, P., Kleinfeld, D., and Linninger, A. (2017). The capillary bed offers the largest hemodynamic resistance to the cortical blood supply. J. Cereb. Blood Flow Metab. 37, 52-68.

Griggs, D., Khateeb, K., Zhou, J., Liu, T., Wang, R., and Yazdan-Shahmorad, A. (2021a). Multimodal artificial dura for simultaneous large-scale optical access and large-scale electrophysiology in non-human primate cortex. J. Neural Eng. 18, 055006. Griggs, D.J., Khateeb, K., Philips, S., Chan, J.W., Ojemann, W., and Yazdan-Shahmorad, A. (2019). Optimized large-scale optogenetic interface for non-human primates. In Optogenetics and Optical Manipulation 2019, S.K. Mohanty, and E.D. Jansen, eds. (SPIE), p. 3.

Griggs, D.J., Belloir, T., and Yazdan-Shahmorad, A. (2021b). Large-scale neural interfaces for optogenetic actuators and sensors in non-human primates. 11663, 1166305.

11 Gulati, T., Won, S.J., Ramanathan, D.S., Wong, C.C., Bodepudi, A., Swanson, R.A., and

12 Ganguly, K. (2015). Robust neuroprosthetic control from the stroke perilesional cortex. J.

13 Neurosci. 35, 8653-8661.

14 Harrison, T.C., Silasi, G., Boyd, J.D., and Murphy, T.H. (2013). Displacement of sensory maps 15 and disorganization of motor cortex after targeted stroke in mice. Stroke 44, 2300-2306.

16 Heffner, H.E., and Heffner, R.S. (1986). Hearing loss in Japanese macaques following bilateral auditory cortex lesions. J. Neurophysiol. 55, 256-271.

Humphrey, N. (1974). Vision in a monkey wihtout striate cortex: a case study. Perception 3, 241-255.

Jiang, W., Gu, W., Hossmann, K.A., Mies, G., and Wester, P. (2006). Establishing a photothrombotic "ring" stroke model in adult mice with late spontaneous reperfusion: Quantitative measurements of cerebral blood flow and cerebral protein synthesis. J. Cereb. Blood Flow Metab. 26, 927-936.

Kaeser, M., Wyss, A.F., Bashir, S., Hamadjida, A., Liu, Y., Bloch, J., Brunet, J.F., Belhaj-Saif, A., and Rouiller, E.M. (2010). Effects of unilateral motor cortex lesion on ipsilesional hand's reach and grasp performance in monkeys: Relationship with recovery in the contralesional hand. J. Neurophysiol. 103, 1630-1645.

Kashani, A.H., Chen, C.L., Gahm, J.K., Zheng, F., Richter, G.M., Rosenfeld, P.J., Shi, Y., and Wang, R.K. (2017). Optical coherence tomography angiography: A comprehensive review of current methods and clinical applications. Prog. Retin. Eye Res. 60, 66-100.

Khateeb, K., Griggs, D.J., Sabes, P.N., and Yazdan-Shahmorad, A. (2019a). Convection enhanced delivery of optogenetic adeno-associated viral vector to the cortex of rhesus macaque under guidance of online MRI images. J. Vis. Exp. 2019.

34 Khateeb, K., Yao, Z., Kharazia, V.N., Burunova, E.P., Song, S., Wang, R., and YazdanCortex of Nonhuman Primates*. Proc. Annu. Int. Conf. IEEE Eng. Med. Biol. Soc. EMBS 35153518.

Khodagholy, D., Gelinas, J.N., Thesen, T., Doyle, W., Devinsky, O., Malliaras, G.G., and Buzsáki, G. (2015). NeuroGrid: Recording action potentials from the surface of the brain. Nat. 
Neurosci. 18, 310-315.

Ledochowitsch, P., Yazdan-Shahmorad, A., Bouchard, K.E., Diaz-Botia, C., Hanson, T.L., He, J.W., Seybold, B.A., Olivero, E., Phillips, E.A.K., Blanche, T.J., et al. (2015a). Strategies for optical control and simultaneous electrical readout of extended cortical circuits. J. Neurosci. Methods 256, 220-231.

Ledochowitsch, P., Yazdan-Shahmorad, A., Bouchard, K.E., Diaz-Botia, C., Hanson, T.L., He, J.W., Seybold, B.A., Olivero, E., Phillips, E.A.K., Blanche, T.J., et al. (2015b). Strategies for optical control and simultaneous electrical readout of extended cortical circuits. J. Neurosci.

9 Methods 256, 220-231.

Li, Y., Wei, W., and Wang, R.K. (2018). Capillary flow homogenization during functional activation revealed by optical coherence tomography angiography based capillary velocimetry.

12 Sci. Rep. 8, 1-9.

Liu, Y., and Rouiller, E.M. (1999). Mechanisms of recovery of dexterity following unilateral lesion of the sensorimotor cortex in adult monkeys. In Experimental Brain Research, (Springer Verlag), pp. 149-159.

Macknik, S.L., Alexander, R.G., Caballero, O., Chanovas, J., Nielsen, K.J., Nishimura, N., Schaffer, C.B., Slovin, H., Babayoff, A., Barak, R., et al. (2019). Advanced Circuit and Cellular Imaging Methods in Nonhuman Primates. J. Neurosci. 39, 8267-8274.

Maeda, M., Takamatsu, H., Furuichi, Y., Noda, A., Awaga, Y., Tatsumi, M., Yamamoto, M., Ichise, R., Nishimura, S., and Matsuoka, N. (2005). Characterization of a novel thrombotic middle cerebral artery occlusion model in monkeys that exhibits progressive hypoperfusion and robust cortical infarction. J. Neurosci. Methods 146, 106-115.

Murata, Y., Higo, N., Oishi, T., Yamashita, A., Matsuda, K., Hayashi, M., and Yamane, S. (2008). Effects of motor training on the recovery of manual dexterity after primary motor cortex lesion in macaque monkeys. J. Neurophysiol. 99, 773-786.

Murphy, A.P., Leopold, D.A., Humphreys, G.W., and Welchman, A.E. (2016). Lesions to right posterior parietal cortex impair visual depth perception from disparity but not motion cues. Philos. Trans. R. Soc. B Biol. Sci. 371.

Nudo, R.J., and Milliken, G.W. (1996). Reorganization of movement representations in primary motor cortex following focal ischemic infarcts in adult squirrel monkeys. J. Neurophysiol. 75, 2144-2149.

Nudo, R.J., Wise, B.M., SiFuentes, F., and Milliken, G.W. (1996). Neural substrates for the effects of rehabilitative training on motor recovery after ischemic infarct. Science (80-. ). 272, 1791-1794.

Nudo, R.J., Larson, D., Plautz, E.J., Friel, K.M., Barbay, S., and Frost, S.B. (2003). A squirrel monkey model of poststroke motor recovery. ILAR J. 44, 161-174. in posterior parietal area 5 in monkeys result in rapid behavioral and cortical plasticity. J.

39 Neurosci. 30, 12918-12935. 
1 Park, K.S., Shin, J.G., Qureshi, M.M., Chung, E., and Eom, T.J. (2018). Deep brain optical coherence tomography angiography in mice: in vivo, noninvasive imaging of hippocampal 3 formation. Sci. Rep. 8, 11614. Paxinos, G., Huang, X.-F., Petrides, M., and Toga, A.W. (2009). The Rhesus Monkey Brain in Stereotaxic Coordinates (San Diego, California, USA).

Pons, T.P., Garraghtyt, P.E., and Mishkin, M. (1988). Lesion-induced plasticity in the second somatosensory cortex of adult macaques.

8 Schaffer, C.B., Friedman, B., Nishimura, N., Schroeder, L.F., Tsai, P.S., Ebner, F.F., Lyden, 9 P.D., and Kleinfeld, D. (2006). Two-photon imaging of cortical surface microvessels reveals a robust redistribution in blood flow after vascular occlusion. PLoS Biol. 4, 258-270.

11 Schmid, M.C., Panagiotaropoulos, T., Augath, M.A., Logothetis, N.K., and Smirnakis, S.M. 12 (2009). Visually Driven Activation in Macaque Areas V2 and V3 without Input from the 13 Primary Visual Cortex. PLoS One 4, e5527.

14 Sommer, C.J. (2017). Ischemic stroke: experimental models and reality. Acta Neuropathol.

15 Teo, L., and Bourne, J.A. (2014). A reproducible and translatable model of focal ischemia in the 16 visual cortex of infant and adult marmoset monkeys. Brain Pathol. 24, 459-474.

17 Tremblay, S., Acker, L., Afraz, A., Albaugh, D.L., Amita, H., Andrei, A.R., Angelucci, A., 18 Aschner, A., Balan, P.F., Basso, M.A., et al. (2020). An Open Resource for Non-human Primate 19 Optogenetics. Neuron 108, 1075-1090.e6.

20 Vallar, G., Rusconi, M.L., Bignamini, L., Geminiani, G., and Perani, D. (1994). Anatomical 21 correlates of visual and tactile extinction in humans: A clinical CT scan study. J. Neurol. 22 Neurosurg. Psychiatry 57, 464-470.

23 Virley, D., Hadingham, S.J., Roberts, J.C., Farnfield, B., Elliott, H., Whelan, G., Golder, J., 24 David, C., Parsons, A.A., and Hunter, A.J. (2004). A New Primate Model of Focal Stroke: Endothelin-1-Induced Middle Cerebral Artery Occlusion and Reperfusion in the Common Marmoset. J. Cereb. Blood Flow Metab. 24, 24-41.

27 WKS, O., DJ, G., Z, I., O, C., H, J., S, M.-C., S, M., and A, Y.-S. (2020). A MRI-Based Toolbox 28 for Neurosurgical Planning in Nonhuman Primates. J. Vis. Exp. 2020, 1-16.

29 Wu, D., Chen, J., Wang, B., Zhang, M., Shi, J., Ma, Y., Zhu, Z., Yan, F., He, X., Li, S., et al. 30 (2016). Endovascular ischemic stroke models of adult rhesus monkeys: A comparison of two 31 endovascular methods. Sci. Rep. 6, 1-10.

32 Wurtz, R.H., and Goldberg, M.E. (1972). Activity of superior colliculus in behaving monkey.

33 IV. Effects of lesions on eye movements. J. Neurophysiol. 35, 587-596.

34 Xerri, C., Merzenich, M.M., Peterson, B.E., and Jenkins, W. (1998a). Plasticity of primary 35 somatosensory cortex paralleling sensorimotor skill recovery from stroke in adult monkeys. J. 36 Neurophysiol. 79, 2119-2148.

37 Xerri, C., Merzenich, M.M., Peterson, B.E., and Jenkins, W. (1998b). Plasticity of Primary 38 Somatosensory Cortex Paralleling Sensorimotor Skill Recovery From Stroke in Adult Monkeys. 
J. Neurophysiol. 79, 2119-2148.

2 Xu, J., Song, S., Wei, W., and Wang, R.K. (2017). Wide field and highly sensitive angiography

3 based on optical coherence tomography with akinetic swept source. Biomed. Opt. Express 8 ,

4420.

5 Yaroslavsky, A.N., Schulze, P.C., Yaroslavsky, I. V., Schober, R., Ulrich, F., and Schwarzmaier,

6 H.J. (2002). Optical properties of selected native and coagulated human brain tissues in vitro in

7 the visible and near infrared spectral range. Phys. Med. Biol. 47, 2059-2073.

8 Yazdan-Shahmorad, A., Diaz-Botia, C., Hanson, T., Ledochowitsch, P., Maharabiz, M.M., and

9 Sabes, P.N. (2015). Demonstration of a setup for chronic optogenetic stimulation and recording

10 across cortical areas in non-human primates. Opt. Tech. Neurosurgery, Neurophotonics,

11 Optogenetics II 9305, 93052K.

12 Yazdan-Shahmorad, A., Diaz-Botia, C., Hanson, T.L., Kharazia, V., Ledochowitsch, P.,

13 Maharbiz, M.M., and Sabes, P.N. (2016). A Large-Scale Interface for Optogenetic Stimulation

14 and Recording in Nonhuman Primates. Neuron 89, 927-939.

15 Yazdan-Shahmorad, A., Silversmith, D.B., Kharazia, V., and Sabes, P.N. (2018a). Targeted

16 cortical reorganization using optogenetics in non-human primates.

17 Yazdan-Shahmorad, A., Tian, N., Kharazia, V., Samaranch, L., Kells, A., Bringas, J., He, J.,

18 Bankiewicz, K., and Sabes, P.N. (2018b). Widespread optogenetic expression in macaque cortex

19 obtained with MR-guided, convection enhanced delivery (CED) of AAV vector to the thalamus.

20 J. Neurosci. Methods. 